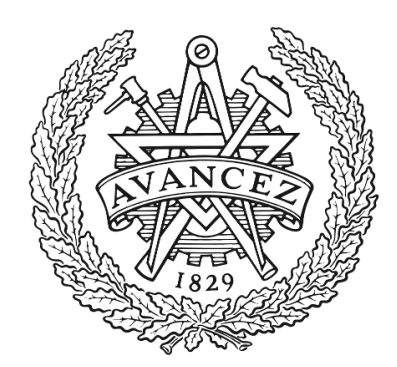

\title{
CHALMERS
}

UNIVERSITY OF TECHNOLOGY

\section{Dispatch Planning of a Wide-Area Wind Power-Energy Storage Scheme Based on Ensemble Empirical Mode Decomposition Technique}

Downloaded from: https://research.chalmers.se, 2023-04-26 10:51 UTC

Citation for the original published paper (version of record):

Li, M., Li, Y., Choi, S. (2021). Dispatch Planning of a Wide-Area Wind Power-Energy Storage Scheme Based on Ensemble Empirical

Mode Decomposition Technique. IEEE Transactions on Sustainable Energy, 12(2): 1275-1288.

http://dx.doi.org/10.1109/TSTE.2020.3042385

N.B. When citing this work, cite the original published paper.

(O2021 IEEE. Personal use of this material is permitted.

However, permission to reprint/republish this material for advertising or promotional purposes 


\title{
Dispatch Planning of a Wide-Area Wind Power-Energy Storage Scheme Based on Ensemble Empirical Mode Decomposition Technique
}

\author{
Mengting Li, Yang Li, Member, IEEE, and San Shing Choi
}

\begin{abstract}
This paper addresses the challenging task of developing a procedure for the day-ahead dispatch planning of wind power which emanates from a wide geographical area. Using the complete ensemble empirical mode decomposition technique, it is shown that the low-frequency components of the area aggregated wind power account for the largest proportions of the perturbing energy harnessed from the wind. By taking advantage of the slow-varying characteristics of the low-frequency components, accurate forecast of these components is readily obtained and incorporated into the developed dispatch planning procedure. The dispatchability of the wide-area wind generation is facilitated by the buffering actions offered by a centralized power dispatch energy storage system, operating under a proposed power flows control strategy. The efficacy of the developed procedure is illustrated using a pumped hydroelectric system as the dispatch energy storage medium.
\end{abstract}

Index Terms-Complete ensemble empirical mode decomposition with adaptive noise (CEEMDAN), dispatch planning, energy storage system, pumped hydroelectric storage, wind power.

\section{INTRODUCTION}

\section{A. Background, Motivation, and Literature Review}

W IND power generation is assuming an increasingly important role in the electricity supply market. By the end of 2019, global installed wind power generating capacity has reached some $622 \mathrm{GW}$ [1]. Unfortunately, the fuel source for the wind turbine generators (WTGs), i.e., the wind, is stochastic and intermittent. As obtaining accurate forecasts of the wind power is challenging, potential contributions from wind farms are often excluded in the conventional power dispatch planning process of grid systems. This practice may be acceptable when the penetration level of the wind generation is relatively low [2]. However, as the rapid growth of wind power generation is expected to persist for the foreseeable future, it will be unwise to continue to exclude the WTG from the dispatch planning process, in view of the significant economic and environmental benefits this renewable generation can bring to society [3].

Mengting Li was with the School of Electrical and Electronic Engineering, Nanyang Technological University, Singapore 639798, Singapore (e-mail: mli1@e.ntu.edu.sg).

Yang Li is with the School of Automation, Wuhan University of Technology, Wuhan 430070, China, and also with the Department of Electrical Engineering, Chalmers University of Technology, 41296 Gothenburg, Sweden (e-mail: yangli@ieee.org).

San Shing Choi is with the School of Electrical Engineering and Robotics, Queensland University of Technology, Brisbane, QLD 4001, Australia (e-mail: sanshing.choi@qut.edu.au).
In general, to participate in short-term dispatch planning under the purview of a competitive electricity market, all participating generating stations must submit dispatch bids to the grid system operator, typically a day or so ahead. The bids would consist of the output power schedules of the generating stations, specified at a regular dispatch interval (DI) which is of the order of 15 to $60 \mathrm{~min}$ [4]. Unfortunately, the uncertain nature of the wind makes it difficult for a wind farm to keep to its bids. A conventional means to compensate for the mismatch between the actual and the scheduled wind generation output powers is to utilize the operating reserves provided by online fossil-fuel generating units. However, this can be an expensive practice.

A more sustainable and promising alternative to the provision of the conventional reserves is to utilize energy storage systems (ESSs). This awareness of the potential use of ESSs leads to a large body of recent works on dispatchable wind farms equipped with centralized ESSs, where the ESS could be in the form of battery energy storage systems (BESSs) [3], [5], [6], superconducting magnetic energy storage systems [7], pumped hydroelectric storage (PHS) systems [8], compressed air energy storage (CAES) systems [9], and power-to-gas [10]. Research attention has also been directed toward the design of hybrid energy storage systems (HESSs) for wind power dispatch planning [11], [12]. In an HESS, different types of ESS with complementary response characteristics are to operate co-operatively, with the objective to meet both the dispatch commitment and the power quality requirements. In these works, the effectiveness of the short-term dispatch schedules relies heavily on the accuracy of the forecast of the power harnessed by the WTGs in the wind farms. In recent years, however, there is a trend toward the development of wide-area wind power generating facilities in many parts of the world. For example, planning is under way to develop $8 \mathrm{GW}$ wind power generation in an area spanning some $1100 \mathrm{~km}^{2}$ in Northwest China. The largest proportion of the generated power is to be transmitted to the eastern regions of the country through an extensive grid system which, at times, has limited transmission capacity [13]. It is therefore necessary to re-examine if a more suitable dispatch planning technique can are found for such a large-scale wind power generation scheme. Due to the vast geographical area involved and the differences in the terrain, the heterogeneity between the WTGs sited over such a large area has to be considered. To obtain accurate forecast of the area wind power will add considerable challenge to the development of the dispatch plan. And yet a 
successful dispatch plan is needed as this will bring immense positive impact to society.

As the first step toward gaining a better understanding of the challenge of wide-area wind power dispatch planning, the outcome of a detailed analysis of the output power of a wind farm will be shown in a latter section. The analysis shows the presence of a number of oscillating wind power components which have periods shorter than, or comparable to, a DI. Over the dispatch planning horizon of up to a day ahead, these relatively high-frequency (HF) wind power components will contribute insignificantly toward the net amount of energy flow from the wind farm. Similar observation has also been drawn in [14]. Hence, it is not necessary to accurately forecast these HF components for the dispatch planning of the wind farm. Instead, ESSs with the capability to respond to the HF wind power components can be utilized to buffer these fastvarying components in order to enhance the quality of the power supply, as has been done in [11]. It is the remaining lowfrequency (LF) oscillating wind power components that need to be considered in the dispatch planning. Since the variations of the LF wind power components would be slower compared with those in the HF components, it will be shown later that accurate forecasts of the LF wind power components can be more readily obtained for the purpose of short-term dispatch planning. Such a distinct advantage will be exploited in the present investigation.

Other researchers have also recognized the important role played by the LF wind power components in the design and operation of wind farms. Their investigations can be broadly categorized into two groups. In the first group, one-step-ahead control techniques, such as those based on first-order lowpass filter [15], Kalman filter [16], and $H_{\infty}$ filter [17], are used to smoothen the fluctuating wind power, often with the view to mitigate the negative impact of the perturbing wind power on the power quality of the grid system. These filtering algorithms rely on the most recent historical wind speed data, and when used in conjunction with other machine learning techniques such as the adaptive linear neuron network, can enhance the one-step-ahead power smoothing effects [18], [19]. The methods determine the real-time wind farm output power and are therefore unsuitable for direct application in dispatch planning whereby the planning horizon can be up to a day ahead.

In the second group, time-frequency analysis techniques are used to analyze and extract the LF components contained in the long-term historical wind speed data. Reported works in this area can in turn be sub-divided into those leading to non-adaptive and adaptive power smoothing methods. Nonadaptive methods include those using discrete Fourier transform (DFT) [20], discrete wavelet transform (DWT) [21], or a combination of both [22]. The main focus of these works is on the design of the ESS in conjunction with WTGs. In general, DFT assumes the data-set under analysis are stationary. However, wind power is intrinsically non-stationary and hence, DFT is less than ideal for use in this analysis. In contrast, DWT is a powerful computational tool for analyzing a non-stationary signal by decomposing the signal into a set of mutually orthogonal wavelet basis functions. The efficacy of the decomposition depends on the carefully selected mother wavelets which, unfortunately, can be difficult to determine in practice. DWT is considered non-adaptive because as the condition under which the signal is produced changes, there is no provision in the DWT to adaptively vary the mother wavelets in response to the change. Indeed, the suitability of DWT for use in developing adaptive short-term dispatch plan has yet to be fully explored.

Recognizing the shortcomings of the non-adaptive power smoothing methods, adaptive methods such as that based on the empirical mode decomposition (EMD) have been proposed for wind power dispatch study [23]. EMD method was developed specifically to analyze nonlinear and non-stationary signals. Based on the local characteristic time scale, EMD technique decomposes the signal into a set of almost orthogonal components known as intrinsic mode functions (IMFs) [24], [25]. The IMFs reflect the natural oscillatory modes embedded in the signal and serve as the basis functions which are determined by the signal itself, rather than by the predetermined kernels, e.g., the mother wavelets in DWT. Several analytical approaches based on EMD have been proposed for wind speed forecasting, focusing mostly over very short-term (from several minutes up to a few hours ahead) forecast horizons [26], [27]. Assuming the highly nonlinear relationship between the wind speed and the WTG output power is known, one could conceivably obtain the very short-term forecast of the WTG output power based on the forecasted wind speed. However, as will be shown in a latter section, WTG output power tends to be intermittent and the application of the EMD analysis technique to it can give rise to the so-called mode mixing and disparate amplitudes issues in the IMFs. This can in turn complicate the determination of the dispatch plan. Also, as shall be shown in a latter section, using the EMD technique to obtain the wind power forecast would require such a high solution time that online day-ahead dispatch planning becomes quite untenable.

\section{B. Major Contributions}

In view of the above, the present investigation aims to develop an analytical dispatch planning methodology for largescale wind power harnessed over a wide area. The major contributions of the work are described as follows.

Firstly, a hierarchical framework of energy storage facility for the wide-area wind generation is proposed. The combined wind power-ESS scheme includes distributed ESSs which are tasked to improve the power quality, and a centralized ESS which is assigned the role of facilitating the dispatchability of the area wind power. The key contribution here is in the approach to demarcate the responsibilities of the distributed and centralized ESSs, based on the frequency and energy contents of the oscillating wind power components.

Secondly, as the primary focus of the present work is on dispatch planning, a two-step method is proposed which yields accurate day-ahead forecast of the LF components in the wind power. The first step is to extract the LF components using the complete ensemble EMD with adaptive noise (CEEMDAN) technique [28]. This new analytical approach is adopted to overcome the issues of mode mixing, disparate amplitudes, 
and high computation burden encountered when using the conventional EMD technique. In the second step, the LF components of the wind power then form the inputs of an artificial neural network (ANN) which is tasked to generate the dayahead forecast of the area LF wind power components. The slow-varying characteristics of the LF wind power components permit the forecast to be accurately produced, and in time for the construction of the day-ahead dispatch plan.

Thirdly, based on the above hierarchical ESS framework and the LF wind power forecast method, the realization of the day-ahead dispatch plan is facilitated by applying a proposed power flow control strategy to the centralized dispatch-oriented ESS. The provision of the buffering actions of the centralized ESS improves the robustness of the area wind power dispatch. Also, by assuming the wind generation enjoys the highest priority on dispatch, the required energy storage capacity of the centralized ESS is shown to be modest, compared to the amount of energy extracted from the WTGs.

\section{Paper Organization}

The rest of the paper is organized as follows. Section II presents the hierarchical framework of the ESS facility and the prevailing power market rules from which the formulation of the dispatch plan is to observe. Brief description of the CEEMDAN algorithm and the outcome of obtaining the LF wind power components from such an analysis are included in Section III. Useful insight is gained from the detailed examination of the LF power components in Section IV, in term of the distinct characteristics contained in these power components, and from which the choice of a suitable form of the centralized dispatch-oriented ESS can be achieved. The detailed operating strategy of the centralized ESS is then developed in Section V. Using data obtained from existing wind power generating locations, examples are given in Section VI to illustrate the efficacy of the proposed dispatch planning procedure. The main findings are included in Section VII.

\section{Wide-Area Wind Power Dispatch Planning}

\section{A. Framework of the Wind Power-Energy Storage Scheme}

To set the scene for the present conceptual study, consider the wide-area wind power generation scheme shown in Fig. 1. The power generation is to emanate from $N$ generating clusters. Each cluster is to contain a number of WTGs and it is formed based on specific criteria, such as physical proximity. The WTG group in the $j$ th cluster can be sufficiently described by the aggregated output power $P_{W, j}(t)$ and the group generating capacity $P_{W, \text { rated }, j}$, for $j \in\{1,2, \cdots, N\}$. These $N$ clusters are interconnected to an external grid system, and $P_{D}(t)$ represents the power dispatched to the grid. It is assumed the internal load demands within the clusters are negligible. Since power flow constraints can exist in the grid which will in turn constraint $P_{D}(t)$, hard dispatch limits shall be placed on $P_{D}(t)$ as the dispatch plan for $P_{D}(t)$ is being developed in latter sections.

As the generating clusters are dispersed within a wide area, all the clustered wind power $P_{W, j}(t)$ will not be perfectly correlated. Notwithstanding the so-called spatial smoothing

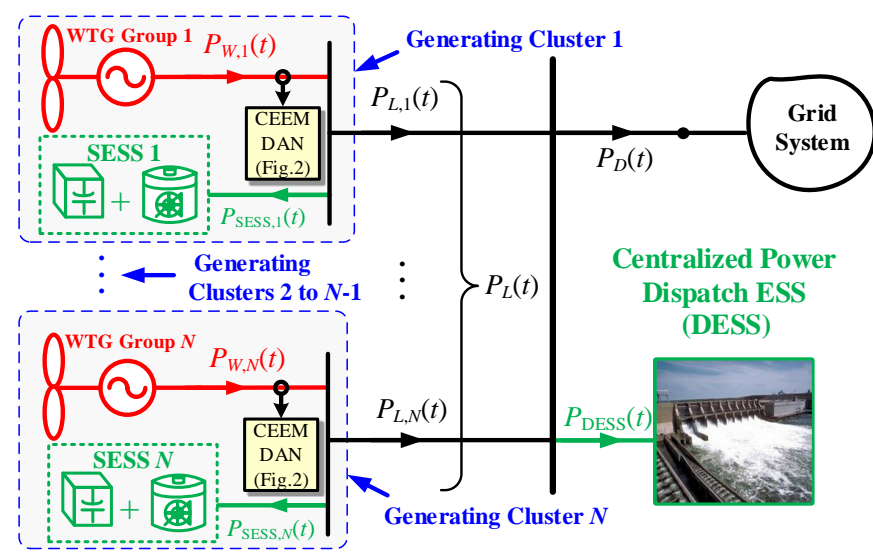

Fig. 1. Conceptual framework of the wide-area wind power generation incorporated with a hierarchical ESS scheme consisting of $\mathrm{N}$ distributed power smoothing ESSs (SESSs) and a centralized power dispatch ESS (DESS). The arrows indicate the reference directions of the power flows.

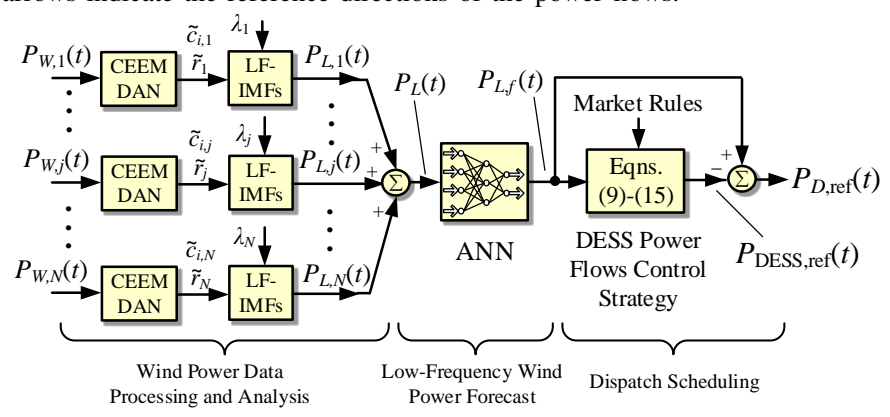

Fig. 2. Schematics of the proposed dispatch planning procedure.

effect [29] which tends to reduce the randomness in the area aggregated wind power $P_{W}(t)=\Sigma P_{W}, j(t)$, direct injection of $P_{W}(t)$ into the grid system could induce unacceptable network voltage and/or frequency deviations. As will be shown in the next section, the non-stationary $P_{W, j}(t)$ can be decomposed into HF and LF components. In view of this and in line with the design considerations given in [11], a local wind power smoothing ESS (denoted as SESS) is to be included in each of the clusters. The SESS in the $j$ th cluster is to buffer the HF wind power components contained in $P_{W, j}(t)$, with the objective to deal with localized power quality issues, such as to ensure that the voltage level of the cluster is within acceptable limits. In Fig. 1, the SESS is shown in the form of a battery-supercapacitor (SC) HESS, although other forms of storage may also be considered. The $j$ th SESS regulates its output power flows $P_{\mathrm{SESS}, j}(t)$ such that the LF net power outflow $P_{L, j}(t)$ from the $j$ th cluster is

$$
P_{L, j}(t)=P_{W, j}(t)-P_{\mathrm{SESS}, j}(t) .
$$

In this way, $P_{L, j}(t)$ will be much smoother than $P_{W, j}(t)$. Next, the aggregation of $P_{L, j}(t)$ in the area yields the area LF wind power component $P_{L}(t)$

$$
P_{L}(t)=\sum_{j=1}^{N} P_{L, j}(t) .
$$

Despite the smoothing actions of the SESS, $P_{L}(t)$ would still be perturbing, albeit at a lower frequency than that seen 
in $P_{W}(t)$. The role of the centralized wind power dispatch ESS, denoted as DESS in Fig. 1, is to buffer $P_{L}(t)$ and to allow the flexible dispatch planning of $P_{D}(t)$.

From Fig. 1, $P_{L}(t)$ is seen as the sum of $P_{D}(t)$ and the power flows $P_{\mathrm{DESS}}(t)$ to the DESS, i.e.,

$$
P_{L}(t)=P_{D}(t)+P_{\mathrm{DESS}}(t) .
$$

A hierarchical arrangement of the ESS has thus been considered in this work whereby the SESS and the DESS are assigned the respective roles of power quality enhancement and dispatch power flows control. Recall the main aim of the present work is on the development of a procedure for the determination of the dispatch plan for $P_{D}(t)$. In the subsequent sections, the details of the various steps involved in the developed procedure shall be given. As shown in Fig. 2, the procedure involves the input of past wind power data $P_{W, j}(t)$ to generate the reference signal $P_{D \text {,ref }}(t)$ for the dispatch plan. It will be shown that $P_{D \text {,ref }}(t)$ can be obtained in time to meet the requirements of the market rules explained in the next subsection. This proposed approach is unlike those reported in the works cited earlier on short-term dispatch planning.

\section{B. Market Rules}

Befitting the status of dispatchable generation and as alluded to earlier, power market rules require the dispatch plan for $P_{D}(t)$ to be submitted to the grid operator $z$ hours ahead. The plan is in the form of a series of power schedules specified at regular DI. The wind power generators are allowed to resubmit revised dispatch plan every $s$ hours, although only those revised schedules for periods not less than $y$ hour(s) ahead can be accepted by the grid operator. Typically, $z=24$, $s=1$, and $y=2$.

In many parts of the world, the impetus is toward achieving low-carbon electricity generation. Consequently, in the present work, it is assumed that the wind generators shall enjoy the highest priority on dispatch and the clusters will be obliged to export all of their generated power to the grid at any given time. Note that if the power market rules were to differ from that assumed above, the proposed dispatch procedure shown in Fig. 2 is still applicable: it only requires the new market rules to be incorporated into the Dispatch Scheduling step shown in the figure. For example, if the wind generators are to participate in a competitive power market, they may submit dispatch bids in order to maximize their incomes. An optimization-based procedure, such as that described in [30], could be used to generate the optimal $P_{D \text {,ref }}(t)$ so as to meet the objective.

\section{ANAlysis OF Wind POWER}

\section{A. Empirical Mode Decomposition of Wind Power Data}

As alluded to in the Introduction section, $P_{W, j}(t)$ pertains to a non-stationary random process and it contains oscillating components over a wide frequency range. EMD technique can be used to analyze such a data-set, as the technique is based on the local characteristic time scale of the data [24], [25]. Using the EMD algorithm, it is shown in [23] that $P_{W, j}(t)$ can be decomposed into $N_{j}$ intrinsic mode functions (IMFs) $c_{i, j}(t)$ plus a residue $r_{j}(t)$,

$$
P_{W, j}(t)=\sum_{i=1}^{N_{j}} c_{i, j}(t)+r_{j}(t)
$$

where $i \in\left\{1,2, \cdots, N_{j}\right\}$ is the order of IMF. For a given $P_{W, j}(t)$, the oscillating frequency of $c_{i, j}$ decreases as the order $i$ increases. Within each $c_{i, j}$, the instantaneous frequency $f_{i, j}(t)$, as defined in [24], is allowed to vary with time. Thus, $P_{W, j}(t)$ is both amplitude- and frequency-modulated by the EMD. In contrast, the residue $r_{j}(t)$ represents the mean trend of the wind power $P_{W, j}(t)$. In the terminology of signal analysis, $P_{W, j}(t)$ tends to be intermittent. The intermittency can lead to the so-called mode mixing and disparate amplitude phenomena [25] when the EMD technique is applied to analyze $P_{W, j}(t)$. To demonstrate these phenomena, examine Figs. 3(a) and (b) which show the wind speed $v_{W, j}(t)$ and the corresponding $P_{W, j}(t)$ of the $j$ th cluster. Expressed in p.u. on $P_{W, \text { rated }, j}$ base, $P_{W, j}(t)$ has been obtained using (5):

$$
P_{W, j}(t)=\left\{\begin{array}{cc}
0, & v_{W, j} \in\left[0, v_{W, \mathrm{ci}, j}\right) \cup\left(v_{W, \mathrm{co}, j}, \infty\right] \\
\left(\frac{v_{W, j}}{v_{W, \text { rated }, j}}\right)^{3}, & v_{W, j} \in\left[v_{W, \mathrm{ci}, j}, v_{W, \text { rated }, j}\right) \\
1, & v_{W, j} \in\left[v_{W, \text { rated }, j}, v_{W, \mathrm{co}, j}\right]
\end{array}\right.
$$

where $P_{W, j}(t)$ assumes a cubic relationship with $v_{W, j}(t)$ when the wind speed is between the WTG cut-in speed $v_{W \text {,ci,j }}$ and the rated speed $v_{W, \text { rated }, j} . P_{W, j}(t)$ is limited to the rated power $P_{W, \text { rated, } j}$ when the wind speed exceeds $v_{W, \text { rated, } j}$ but below the WTG cut-off speed $v_{W, \text { co }, j}$. It can be observed from Fig. 3(b) that $P_{W, j}(t)$ exhibits the intermittency characteristics over the interval 31st -47 th hours when $P_{W, j}(t)$ remains at the maximum level of 1 p.u. as the wind speed is at or above $v_{W, \text { rated }, j}$. Fig. 3(c) shows the corresponding IMFs $c_{i, j}$ for $i=1,3,5$, and 7 as well as the residue $r_{j}(t)$. Mode mixing can be seen in, for example, $c_{1, j}(t)$ over the 31 st -47 th hours period when $P_{W, j}(t)$ is bounded to 1 p.u.: $c_{1, j}(t)$ oscillates at relatively large magnitude and low frequency compared to that outside this period. Such disparate amplitude and mode mixing phenomena complicate the analysis of $P_{W, j}(t)$.

\section{B. Wind Power Analysis Using CEEMDAN}

In order to mitigate the disparate amplitude and mode mixing problems, the CEEMDAN algorithm [28] shall be used in the present investigation to analyze $P_{W, j}(t)$. In this algorithm, the original signal under investigation is artificially modified by adding a series of realizations of white Gaussian noise so that the effectiveness and the computational efficiency of the decomposition process can be significantly improved, compared to the EMD technique. CEEMDAN has been successfully applied in diverse fields of research, including that of distribution system fault detection [31], biomedical image processing [32], traffic flow forecast [33], and medical diagnosis [34]. The computational steps involved are as follows, where the subscripts " $W$ " and " $j$ " of $P_{W, j}(t)$ will be omitted for better readability, and subscript $k$ is attached to indicate the stage in the CEEMDAN algorithm. 

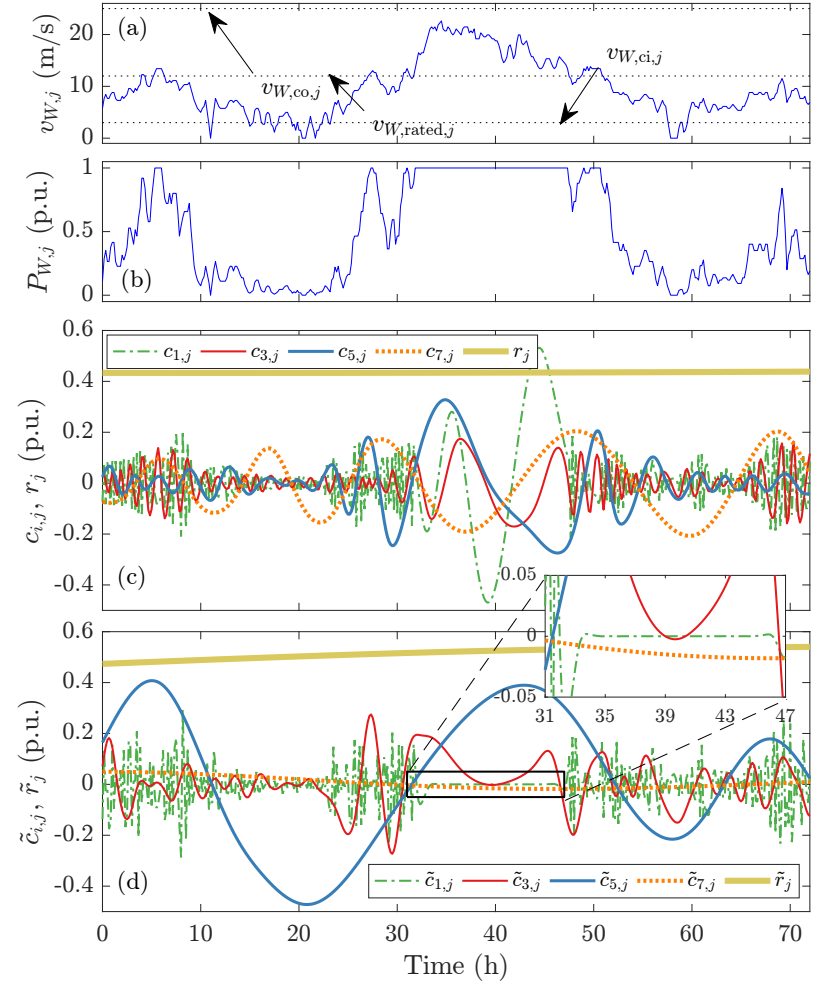

Fig. 3. Sample of (a) wind speed $v_{W, j}(t)$, (b) the corresponding wind power $P_{W, j}(t)$, (c) IMFs $c_{i, j}(t)$ and residue $r_{j}(t)$ obtained from the decomposition of $P_{W, j}(t)$ using the conventional EMD, (d) IMFs $\tilde{c}_{i, j}(t)$ and residue $\tilde{r}_{j}(t)$ obtained from the decomposition of $P_{W, j}(t)$ using CEEMDAN.

Step 1: Initialization $(k=0)$. Add $M$ realizations of white Gaussian noise signals $w^{m}(t)$ to the original signal $P(t)$, to create an ensemble of noisy signals $P_{0}^{m}(t)=P(t)+\varepsilon_{0} w^{m}(t)$. $m \in\{1,2, \cdots, M\}$ is the index of the noise realization, and $\varepsilon_{0}$ is a coefficient that determines the signal-noise ratio. Next, decompose each of $P_{0}^{m}(\mathrm{t})$ by the conventional EMD technique and obtain the first EMD mode $c_{1}^{m}(t)$. Then set

$$
\tilde{c}_{1}(t)=\frac{1}{M} \sum_{m=1}^{M} c_{1}^{m}(t) .
$$

Step 2: First stage $(k=1)$. Calculate the first residue

$$
\tilde{r}_{1}(t)=P(t)-\tilde{c}_{1}(t) .
$$

Step 3: Denote $\mathbb{E}_{n}[\cdot]$ as the operator used to obtain the $n$th EMD modes of [.]. Decompose the realizations $\tilde{r}_{1}^{m}(t)=$ $\tilde{r}_{1}(t)+\varepsilon_{1} \mathbb{E}_{1}\left[w^{m}(t)\right]$ to obtain the first EMD modes for all $m$, and then define the second CEEMDAN IMF as:

$$
\tilde{c}_{2}(t)=\frac{1}{M} \sum_{m=1}^{M} \mathbb{E}_{1}\left[\tilde{r}_{1}^{m}(t)\right] .
$$

Step 4: For $k=2,3, \cdots$, calculate the $k$ th residue by

$$
\tilde{r}_{k}(t)=\tilde{r}_{k-1}(t)-\tilde{c}_{k}(t) \text {. }
$$

Step 5: Decompose the realizations $\tilde{r}_{k}^{m}(t)=\tilde{r}_{k}(t)+$ $\varepsilon_{k} \mathbb{E}_{k}\left[w^{m}(t)\right]$ to obtain their first EMD modes and define the $(k+1)$ th CEEMDAN IMF as:

$$
\tilde{c}_{k+1}(t)=\frac{1}{M} \sum_{m=1}^{M} \mathbb{E}_{k}\left[\tilde{r}_{k}^{m}(t)\right] .
$$

Repeat Steps 4 and 5 until it is no longer feasible to decompose the obtained residue $\tilde{r}_{k}(t)$.

Again, as an illustration, the result of the decomposition based on the CEEMDAN technique is presented in Fig. 3(d) when the technique is applied to analyze the same $P_{W, j}(t)$ data-set shown in Fig. 3(b). It can be readily seen that over the 31 st -47 th hours period when $P_{W, j}(t)$ is bounded to 1 p.u., the amplitude of the first IMF $\tilde{c}_{1, j}(t)$ has been reduced to almost zero. Indeed, each of the IMFs now assumes a relatively more uniform amplitude and occupies a narrower frequency range. It thus indicates that CEEMDAN is able to mitigate the issues of the disparate amplitude and mode mixing in the IMFs. Accordingly, CEEMDAN has been incorporated as the first step in the wind power data processing and analysis stage of the proposed dispatch planning procedure shown in Fig. 2. Also, the CEEMDAN algorithm is much more computationally efficient than EMD [28]. For example, using the Intel Core 2 Q9400 @ $2.67 \mathrm{GHz}$ processor, it requires less than $5 \mathrm{~s}$ to obtain all the IMFs and the residue function for a one-year $P_{W, j}(t)$ data-set, sampled at every $10 \mathrm{~min}$, whereas analyzing the same data-set shall take more than 4 min when EMD is used instead.

\section{Perturbing Energy Levels in the IMFs}

From the outcome of the CEEMDAN analysis, further insights can be gained by examining the changes $\Delta E_{i, j}(t)$ and $\Delta E_{r, j}(t)$ in the energy levels of $\tilde{c}_{i, j}(t)$ and $\tilde{r}_{j}(t)$ respectively. The changes, with respect to the energy levels of $\tilde{c}_{i, j}(t)$ and $\tilde{r}_{j}(t)$ at the initial time of the data-set $P_{W, j}(t)$, can be obtained by integrating $\tilde{c}_{i, j}(t)$ and $\tilde{r}_{j}(t)$ with respect to time. As indicated in Fig. 4(a), the energy levels contained in $\tilde{c}_{i, j}(t)$ also oscillate. $\Delta E_{1, j}(t)$ and $\Delta E_{3, j}(t)$ are examples of the perturbing energy components which are of relatively high frequency but of low magnitudes. Their corresponding IMFs are referred to as high-frequency IMFs (HF-IMFs) in this work. In contrast, $\Delta E_{5, j}(t)$ and $\Delta E_{7, j}(t)$ in Fig. 4(a) are examples of larger oscillating energies in the low-frequency IMFs (LF-IMFs) $\tilde{c}_{5, j}(t)$ and $\tilde{c}_{7, j}(t)$, respectively.

In general, the magnitudes of the oscillating energies in the LF-IMFs are larger than those in the HF-IMFs. This is as expected because the periods of the oscillations in the LFIMFs are larger than those in the HF-IMFs. Although the levels of the oscillating energies in the HF-IMFs are relatively smaller, these energies have to be dealt with, preferably at the generating cluster level, in order to maintain acceptable power quality. This necessary task is assigned to the SESS, as pointed out in Section II. Next, since $\tilde{r}_{j}(t)$ is a positive trend function, $\Delta E_{r, j}(t)$ is an increasing function. This is also to be expected because the cluster is a net exporter of energy. In view of these observations and recall the objective of this work is on developing a procedure on dispatch planning, henceforth the focus shall be on the LF-IMFs and the residue. 

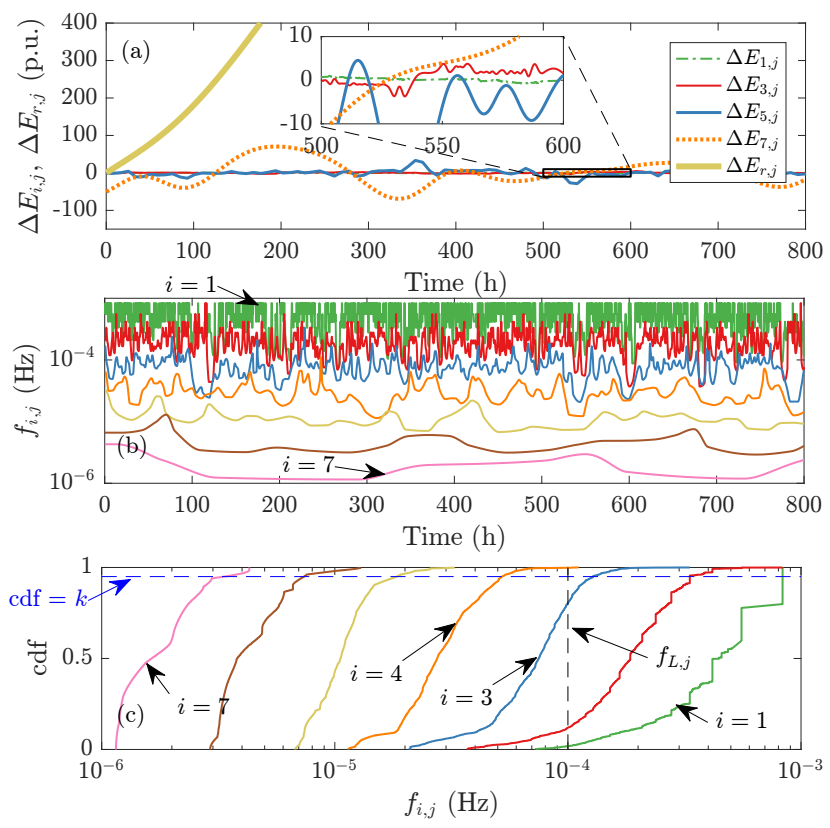

Fig. 4. (a) Changes in the energy levels of IMFs $\tilde{c}_{i, j}(t)$ and residue $\tilde{r}_{j}(t)$ : $\Delta E_{i, j}$ and $\Delta E_{r, j}$. (b) Instantaneous frequencies $f_{i, j}(t)$. (c) cdf of $f_{i, j}(t)$.

Finally, although detailed results have not been included in this manuscript, additional CEEMDAN analysis had been carried out on $P_{W, j}(t)$ which had been sampled at the higher sampling rate of $1 \mathrm{~min} / \mathrm{sample}$. The intent is to examine the energy levels contained in those wind power components which have frequencies higher than $f_{1, j}(t)$, which is shown in Fig. 4(b), i.e., the frequency of the first IMF $\tilde{c}_{1, j}(t)$ discussed earlier. Again, it has been observed that the oscillating energy levels in these HF-IMFs are even lower than the $\Delta E_{1, j}(t)$ shown in Fig. 4(a). Hence, the 10-min sampling rate is considered suitable for the present study on dispatch planning and will be used in the remaining part of this work.

\section{Determination of LF-IMFs}

In the context of the hierarchical ESS system shown in Fig. 1, it has been explained in Section II-A that the role of the DESS is to effect the dispatchability of the largescale wind generation. It would be most desirable if the LFIMFs and the HF-IMFs can be readily identified because the buffering actions of the SESS and that of the DESS can then be considered separately. In this way and in the planning stage of the storage system, the design of the SESS can be carried out without the need to consider the actions of the DESS and vice versa. As the SESS installations are dispersed within the area, in the form of distributed storage, each SESS can be designed in conjunction with its respective cluster of the WTG, whereas the DESS is a centralized storage facility designed to cater for the whole area.

Since each of the IMFs can be related to its frequency, the above approach to segregate the IMFs could be viewed equivalently as the setting of a cutoff frequency, denoted herewith as $f_{L, j}(t)$. The LF-IMFs would have frequency components below $f_{L, j}(t)$. Unfortunately, in practice, it may not be possible to determine the ideal value of $f_{L, j}(t)$ such that the buffering actions of the two groups of IMFs can be completely decoupled over the whole study period. One could attempt to find a value for $f_{L, j}(t)$ which would lead to the minimum interacting actions between the SESS and DESS, an approach which has been proposed in [23]. In [23], the focus is to segregate the power quality enhancement actions of the battery from that of the SC in a hybrid ESS. In the context of the present investigation, the same minimum interacting actions concept may still be used so that the IMFs can be segregated into the LF-IMF and HF-IMF groups. Unfortunately, the approach described in [23] is based on the results of EMD analysis where, as explained in Section III-A, mode mixing between the IMFs is common. This would complicate the task of the segregation of the IMFs.

On the other hand, Section III-B has shown that the CEEMDAN technique is able to mitigate the mode mixing phenomenon. Therefore, an improved empirical method to identify the LF-IMFs will now be described. Recall the dispatch plan is needed so that the grid operator has ample time to arrange for spinning reserve in response to any changes in the output power schedules of the wind power generators. And the present investigation is concerned with the use of the DESS to replace the conventional spinning reserve in buffering the LF-IMFs. So for the DESS to be effective, one has to take into consideration the operational characteristics of the DESS. For example, if the DESS is designed to operate with a chargedischarge cycle time of, say, no less than $3 \mathrm{~h}$ per cycle, this can be translated as the DESS operating at cycle frequency of no more than $1 \times 10^{-4} \mathrm{~Hz}$. Next refer to Fig. 4(b) where it is shown the instantaneous frequencies $f_{i, j}(t)$ of the IMFs obtained from the outcome of the CEEMDAN analysis of the wind power. Each of the $f_{i, j}(t)$ is seen to occupy a frequency band and a quantitative way to describe the time-varying characteristics of the $f_{i, j}(t)$ is to generate its cumulative distribution function (cdf). This is shown in Fig. 4(c). In this work and in consideration of the threshold cycle frequency of $1.0 \times 10^{-4} \mathrm{~Hz}$, an IMF would be considered an LF-IMF if the probability of its frequency $f_{i, j}(t)$ having a value at or below $1.0 \times 10^{-4} \mathrm{~Hz}$ is to exceed a pre-specified threshold level $k$, and $k$ would be the confidence-level design parameter. So unlike the approach of [23], the present work proposes the determination of the number of IMFs, denoted as $\lambda_{j}$, within the set of the LF-IMFs. Again using Fig. 4(b) as an example, there are a total of 7 IMFs obtained as the result of the CEEMDAN analysis. Suppose the threshold $k$ is set at 0.95 . From Fig. 4(c), it is noted that some $99.8 \%$ of the instantaneous frequency $f_{4, j}(t)$ of the 4 th $\operatorname{IMF} \tilde{c}_{4, j}(t)$ is at or below $1.0 \times 10^{-4} \mathrm{~Hz}$ whereas that of $f_{3, j}(t)$ of the next lower order IMF $\tilde{c}_{3, j}(t)$ is 0.8 which is less than $k$. Hence, $\tilde{c}_{4, j}(t)$ to $\tilde{c}_{7, j}(t)$ are members of the set of the LF-IMFs and so $\lambda_{j}=4$.

In view of the above, in the second step of the wind power data processing and analysis stage shown in Fig. 2, $\tilde{c}_{i, j}(t)$ and $\tilde{r}_{j}(t)$, obtained following the CEEMDAN analysis of $P_{W, j}(t)$, and $\lambda_{j}$, are inputted to the $j$ th LF-IMF block. Based on $\lambda_{j}$, the LF-IMFs are extracted among the IMFs $\tilde{c}_{i, j}(t)$ and the 


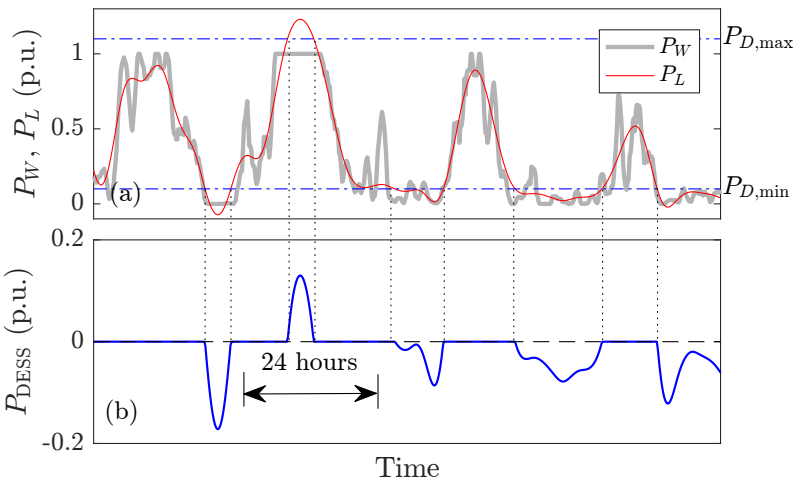

Fig. 5. Example of (a) $P_{W}(t)$ and the LF power component $P_{L}(t)$ obtained from the aggregation of $P_{L, j}(t)$, and (b) the power flows to the DESS.

signal corresponding to $P_{L, j}(t)$ is constructed as

$$
P_{L, j}(t)=\sum_{i=N_{j}-\lambda_{j}+1}^{N_{j}} \tilde{c}_{i, j}(t)+\tilde{r}_{j}(t) .
$$

\section{Power Dispatch Energy Storage System}

\section{A. Characteristics of $P_{L}(t)$ and DESS Operational Strategy}

In this section, the technique to obtain the LF wind power forecast and the development of a DESS power flows control strategy shall be described. The forecast and strategy are needed in the remaining two stages of the dispatch planning procedure shown in Fig. 2.

The previous section shows that $P_{L, j}(t)$ can be readily generated using the CEEMDAN technique. Whence using (2), the area LF wind power component $P_{L}(t)$ can also be determined. While the functions of the DESS shall be explained and demonstrated in greater detail shortly, it will be instructive to highlight some of the interesting characteristics of $P_{L}(t)$ by referring to Fig. 5(a). The figure shows a typical waveform of $P_{L}(t)$, expressed in p.u. on the power base equals to the sum of the power ratings of all the WTGs in the $N$ generating clusters. Notice that $P_{L}(t)$ is considerably smoother than the area aggregated wind power $P_{W}(t)$ and is within 0 and 1.0 p.u. in most instances.

Also shown in Fig. 5(a) are the maximum and minimum allowable dispatch levels $P_{D, \max }$ and $P_{D \text {, min }}$ respectively. In practice, $P_{D, \max }$ and $P_{D, \min }$ are set in accordance with the prevailing grid operational constraints, as alluded to in Section II-A. For example, in areas of rich wind resource but of limited transmission capacity such as in the northwestern China, $P_{D, \max }$ can conceivably be less than 1 p.u. Also as explained in Section II-B, it is reasonable to assume that the wind generators shall enjoy the highest priority on dispatch and the clusters will be obliged to export all of their generated power to the grid at all times. Due to the relatively slower oscillating characteristics of $P_{L}(t), P_{L}(t)$ can be more accurately predicted over the day-ahead dispatch planning horizon. This approach is preferable to that shown in [5] in which the complete $P_{W}(t)$ has to be forecasted. In view of this, it is proposed that the day-ahead dispatch power schedules on $P_{D}(t)$ are set equal to the forecast of $P_{L}(t)$. The forecast on
$P_{L}(t)$ can then be treated as the reference signal for $P_{D}(t)$. Based on the power flows relationship (3), it is clear that the DESS is to buffer the difference between $P_{D}(t)$ and $P_{L}(t)$. However, due to the constraints placed on $P_{D}(t)$, the following scenarios can arise:

Scenario 1: When $P_{D, \min } \leq P_{L}(t) \leq P_{D, \max }$, as can be seen from Fig. 5(a), $P_{L}(t)$ would be within the allowable dispatch levels under this scenario in most instances. Thus by setting $P_{D}(t)=P_{L}(t)$ and in view of $(3), P_{\text {DESS }}(t)=0$. So the DESS would often be idle.

Scenario 2: When $P_{L}(t) \leq P_{D \text {, min }}$, the dispatch power schedules on $P_{D}(t)$ would be set to the minimum dispatch level $P_{D, \text { min }}$. In which case, the DESS will be in the discharging mode and from (3), $P_{\mathrm{DESS}}(t)=P_{L}(t)-P_{D \text {,min }}$.

Scenario 3: For those instances when $P_{L}(t)>P_{D, \max }$, the dispatch power schedules on $P_{D}(t)$ would be set to the maximum dispatch level $P_{D, \max }$. Whence the DESS will be in the charging mode and from (3), $P_{\mathrm{DESS}}(t)=P_{L}(t)-P_{D, \text { max }}$.

An important observation from the above analysis pertains to the amount of DESS stored energy needed to enable the wind power dispatchability. Scenarios 2 and 3 show that the DESS will be in the non-idle state when $P_{L}(t)<P_{D \text {,min }}$ and when $P_{L}(t)>P_{D, \max }$. From Fig. 5(b) and provided the power dispatch limits $P_{D, \text { max }}$ and $P_{D, \text { min }}$ are reasonable, it can be concluded that the DESS shall only be involved in the occasional charging or discharging of a relatively small amount of energy, in comparison to that harnessed from the wind. Therefore, by assigning the highest priority to dispatch the harnessed wind power and the strategy to set the reference signal for $P_{D}(t)$ in accordance with $P_{L}(t)$, it will only require the occasional service of a DESS of modest storage capacity. This is an encouraging development as it would mean a more economically viable DESS.

\section{B. PHS as a Dispatch Energy Storage System}

Having explained the characteristics of $P_{L}(t)$ and the possible operational modes of the DESS, the next task is to develop the control strategy for the DESS so as to affect the wind power dispatchability. As can be seen from Fig. 5(b), the typical duration during which the DESS operates will be more than several hours. Based on the current level of technology, PHS, CAES, and electrochemical batteries can be suitable to assume the role of the DESS. In terms of cost per $\mathrm{kWh}$ storage capacity, CAES and batteries are not as economical as the PHS to sustain the dispatch operation over such extended periods [35]. Although finding a suitable site for the PHS can be challenge and constructing a PHS is subject to environmental regulations, it is nevertheless based on a mature storage technology [36] and has performance characteristics, such as cycle time and speed of response, commensurate with that of $P_{\mathrm{DESS}}(t)$ shown in Fig. 5. Hence, PHS is technically viable and accordingly, it has been selected as the form of the DESS in this work. The development of the control strategy for the PHS shall be described in the next section. It must be understood, however, that the developed strategy will be equally applicable should another suitable type of ESS were to be selected instead. 
With regard to the PHS, the water level in the PHS reservoir reflects the amount of stored energy in the ESS [8], [37]. When the water level is at the maximum designed height, the PHS is considered to be at the maximum stored energy state and the corresponding state-of-energy (SOE) is 1. Conversely, when the water level drops to the minimum designed height, the SOE of the PHS is $1-d_{P, \max }$ where $d_{P, \max }$ denotes the maximum allowable depth of discharge. In this work, the SOE is to be maintained at or near to a target value, denoted as SOE$^{*}$, when the PHS is in the idle mode. This is because apart from functioning as an energy buffer to enable wind power dispatchability, the PHS may also assume a role in irrigation, navigation, among other purposes, whereby it is desirable the water level in the reservoir is kept at an acceptable level under most instances.

\section{Determination of Short-Term Dispatch Plan}

In this section, the technique to obtain the LF wind power forecast and the development of a DESS power flows control strategy shall be described. The forecast and strategy are needed in the remaining two stages of the dispatch planning procedure shown in Fig. 2.

\section{A. Forecast of the Low-Frequency Wind Power}

In the previous two sections, it is shown that the area LF wind power component $P_{L}(t)$ can be readily generated from $P_{W, j}(t)$. With $P_{L}(t)$ known and as explained in Section IV-A, accurate short-term day-ahead forecast $P_{L, f}(t)$ of $P_{L}(t)$ can be obtained based on past $P_{L}(t)$. In this work, selected forecasting techniques described in [38] had been applied to yield $P_{L, f}(t)$. The back-propagation ANN technique was found to perform most satisfactorily in terms of accuracy and solution time. Interested readers may refer to [39] for a general description of ANN. For the problem at hand, the construction of the ANN model involves the use of historical data of $P_{L}(t)$ for the offline setting up and training of the neural network. The obtained ANN model, shown in Fig. 2, consists of the input, hidden, and output layers. The numbers of neurons in the layers are 20,10, and 1, respectively. The sampling rate is $10 \mathrm{~min}$. The well-trained neural network can then be used in the dispatch planning procedure to yield $P_{L, f}(t)$ using the real-time generated function $P_{L}(t)$ as input. Typically, $25 \mathrm{~s}$ is required to generate a 24-h length of $P_{L, f}(t)$ using the processor Intel Core 2 Q9400 @ $2.67 \mathrm{GHz}$. This solution time does not include the time needed for the CEEMDAN analysis of $P_{W, j}(t)$, as such a task shall be carried out in a distributed manner at each cluster location. Nevertheless, the total solution time needed to obtain $P_{L, f}(t)$ using $P_{W, j}(t)$ as the input is clearly much shorter than the dispatch schedule re-submission interval of $s$ hours. Hence, there is ample time for $P_{L, f}(t)$ to be generated for the re-submission.

\section{B. Determination of the Dispatch Plan Reference Signal}

With $P_{L, f}(t)$ generated, the next step is to obtain the reference signal $P_{D \text {,ref }}(t)$ for the dispatch planning. In order to

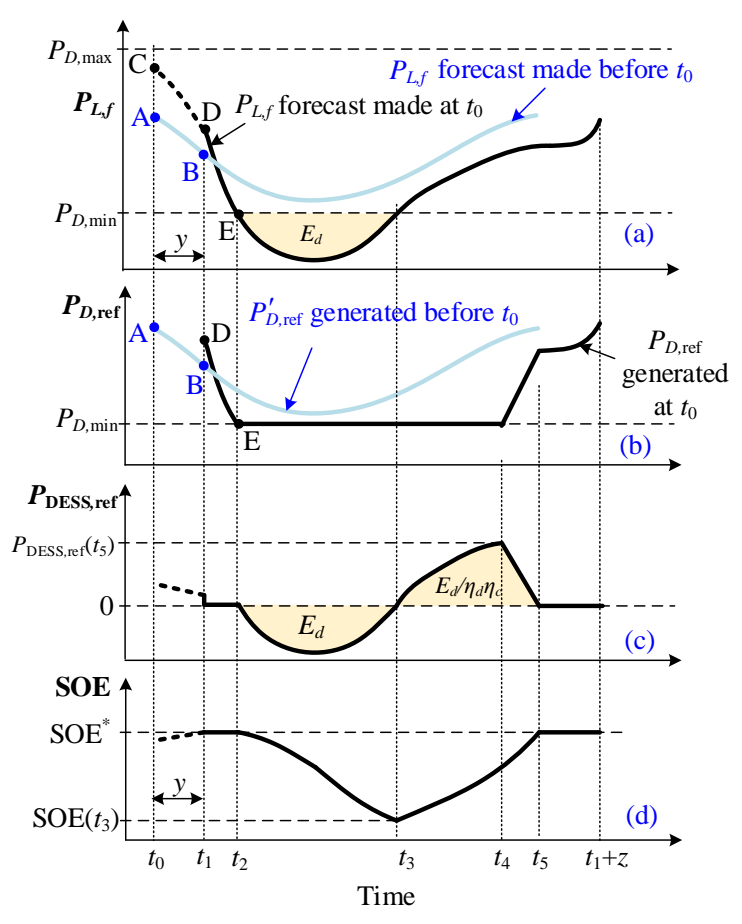

Fig. 6. Example of (a) $P_{L, f}(t)$ determined at and before $t_{0}$, (b) strategized $P_{D \text {,ref }}(t)$ for $t_{1} \leq t \leq t_{1}+z$ determined at $t_{0}$, (c) strategized $P_{\text {DESS,ref }}(t)$ for $t_{1} \leq t \leq t_{1}+z$ determined at $t_{0}$, and (d) SOE for $t_{1} \leq t \leq t_{1}+z$ estimated at $t_{0}$.

do so, the DESS power reference $P_{\mathrm{DESS} \text {,ref }}(t)$ has to be known because from (3),

$$
P_{D, \text { ref }}(t)=P_{L, f}(t)-P_{\text {DESS,ref }}(t)
$$

From Section IV-A, it is clear $P_{\mathrm{DESS}, \mathrm{ref}}(t)$ will be impacted by the market rules as well as the assumption that the wind power generators are to enjoy the highest priority in power dispatch. So in Fig. 2, with $P_{L, f}(t)$ as input, the DESS Power Flows Control Strategy block is to generate $P_{\mathrm{DESS} \text {,ref }}(t)$ based on the principle to be explained herewith.

With reference to Fig. 6, suppose the wind power generation operators are to provide the $z$-hour ahead dispatch plan at time $t_{0}$. As indicated in Fig. 6(a), the forecast $P_{L, f}(t)$ made at $t_{0}$ is shown as the solid black line, whereas that determined before $t_{0}$ is shown as the solid grey line. However, in line with the adopted market rules explained earlier, $P_{D \text {,ref }}(t)$ within the immediate y hours after $t_{0}$ must retain the dispatch schedules based on $P_{L, f}(t)$ made before $t_{0}$, i.e., the curve $\mathrm{A}-\mathrm{B}$ in Fig. 6(b). Therefore, at $t_{0}$, only those revised $z$-hour schedules (solid black line) corresponding to the period $t_{1}=t_{0}+y$ to $t_{1}+z$ will be accepted by the grid operator. Accordingly, $P_{D \text {,ref }}(t)$ over the period $t_{1}$ to $t_{1}+z$ will be determined, in the manner illustrated in Fig. 6(a) and Fig. 6(b) where a Scenario 2 event alluded to in Section IV-A occurs, as follows.

Over the interval $t_{1} \leq t \leq t_{2}$, Fig. 6(a) shows that $P_{L, f}(t) \geq P_{D \text {,min }}$, i.e., the predicted LF power component aggregated from the $N$ wind power generating clusters is higher than $P_{D \text {, min }}$. As will be shown later, the SOE of the PHS shall be close to its target value $\mathrm{SOE}^{*}$ at this stage of the PHS operations. Also, as $P_{L, f}(t)$ is within the allowable dispatch 
levels and the wind generators have the highest priority in power dispatch, it is necessary to export all the outflow power to the grid, i.e., $P_{D, \text { ref }}=P_{L, f}$. From (8), therefore, the PHS power reference $P_{\mathrm{DESS}, \mathrm{ref}}(t)$ shall be 0 ,

$$
\left.\begin{array}{c}
P_{\mathrm{DESS}, \mathrm{ref}}(t)=0 \\
P_{D, \mathrm{ref}}(t)=P_{L, f}(t)
\end{array}\right\} \text { for } t_{1} \leq t \leq t_{2}
$$

Based on the forecast $P_{L, f}(t)$ made at $t_{0}, P_{L, f}(t)$ is predicted to have reached the $P_{D \text {,min }}$ level at $t=t_{2}$, as shown in Fig. 6(a). Since the SOE of the PHS can be tracked in realtime, thus the SOE of the PHS at $t_{0}$ is known and so the SOE of the PHS at $t_{2}$ can be estimated as

$$
\begin{aligned}
\operatorname{SOE}\left(t_{2}\right) & =\operatorname{SOE}\left(t_{0}\right)+\frac{1}{E_{P, r}} \int_{t_{0}}^{t_{1}}\left(P_{L, f}(t)-P_{D, \text { ref }}^{\prime}(t)\right) d t \\
& +\frac{1}{E_{P, r}} \int_{t_{1}}^{t_{2}}\left(P_{L, f}(t)-P_{D, \text { ref }}(t)\right) d t
\end{aligned}
$$

In (10), $E_{P, r}$ denotes the rated energy storage capacity of the PHS, while $P_{D \text {,ref }}$ and $P_{D \text {,ref }}^{\prime}$ represent the dispatch power reference signals obtained at $t_{0}$ and before $t_{0}$, respectively.

Also in (10) and as alluded to earlier, over the $y$-hours interval $t_{0} \leq t \leq t_{1}, P_{D, \text { ref }}(t)$ is set equal to the forecast $P_{L, f}(t)$ made before $t_{0}$, as reflected by the curves A-B in Fig. 6(a) and Fig. 6(b). However, the forecast $P_{L, f}(t)$ generated at $t_{0}$ will inevitably differ from that made before $t_{0}$. The dotted curve C-D in Fig. 6(a) shows the portion of $P_{L, f}(t)$ generated at $t_{0}$ over the interval $t_{0}<t<t_{1}$. The PHS acts to compensate for the difference between the two forecasts $\mathrm{A}-\mathrm{B}$ and $\mathrm{C}-\mathrm{D}$ over the $y$ hours. This will cause an expected small change in the SOE of the PHS. This effect is reflected by the second term on the RHS of (10). On the other hand, since over the interval $t_{1} \leq t \leq t_{2}, P_{D, \text { ref }}(t)=P_{L, f}(t)$ or $P_{\text {DESS,ref }}(t)=0$, the trajectories of $P_{D, \text { ref }}(t)$ and $P_{L, f}(t)$ will be the curve D-E in Fig. 6(a) and Fig. 6(b), respectively. Hence, the third term on the RHS of (10) will be zero.

Next, over the interval $t_{2} \leq t \leq t_{3}$, Fig. 6(a) shows that a new situation has occurred as $P_{L, f}(t)$ is lower than $P_{D \text {,min }}$. As alluded to under Scenario 2 in Section IV-A, so $P_{D \text {,ref }}(t)$ is set equal to $P_{D, \text { min }}$, and the shortfall $P_{D, \text { min }}-P_{L, f}(t)$ is to be supplied by the DESS. So the DESS is called upon to discharge, as shown in Fig. 6(c)

$$
\left.\begin{array}{l}
P_{\mathrm{DESS}, \text { ref }}(t)=P_{L, f}(t)-P_{D, \text { min }} \\
P_{D, \text { ref }}(t)=P_{D, \text { min }}
\end{array}\right\} \text { for } t_{2} \leq t \leq t_{3} .
$$

The amount of the discharged energy is equal to $E_{d} / \eta_{d}$, where $\eta_{d}$ is the efficiency of the PHS discharging process. $E_{d}$ is the energy corresponding to the shaded area shown in Fig. 6(a). $E_{d}$ can be readily determined since $P_{L, f}(t)$ is known. Whence, the SOE of the PHS at the end of the discharging process at $t_{3}$ can be estimated as

$$
\operatorname{SOE}\left(t_{3}\right)=\operatorname{SOE}\left(t_{2}\right)-E_{d} /\left(\eta_{d} E_{P, r}\right) .
$$

Beyond $t_{3}$, Fig. 6(a) shows that $P_{L, f}(t)$ is predicted to be above $P_{D \text {,min }}$ again. Since the PHS has been discharging over the interval $t_{2} \leq t \leq t_{3}, \operatorname{SOE}\left(t_{3}\right)$ will be less than SOE* Instead of exporting all $P_{L, f}(t)$ to the grid, it will be prudent to direct the surplus $P_{L, f}(t)-P_{D \text {,min }}$ to re-charge the PHS so that the PHS can reach the target SOE level SOE* in the shortest possible time. This will maximize the probability the PHS would then have the appropriate amount of stored energy to carry out its next discharge/charge duty.

As the PHS re-charges, the SOE of the PHS is expected to be close to its target value $\mathrm{SOE}^{*}$ at $t=t_{4}$. So it will then be desirable to institute a smooth ramping-down charging strategy on $P_{\mathrm{DESS}, \text { ref }}(t)$. This is to be accomplished at a rate in compliance with the maximum allowable power ramp rate stipulated in grid code, such as that given in [40], and/or that permissible for the safe ramping-down operation of the PHS, whichever is applicable. This maximum allowable power ramp rate is denoted as $\gamma_{\max }$. It is proposed herewith that $P_{\mathrm{DESS}, \text { ref }}(t)$ is to ramp down at the constant rate $\gamma_{\max }$. By numerical means, one can readily determine the time $t_{4}$ when the charging process should start to ramp down at the maximum rate of max such that by the time $t_{5}$, the SOE of the PHS is SOE* The shaded area in Fig. 6(c) shown bounded by $P_{\mathrm{DESS}, \text { ref }}$ for $t_{3} \leq t \leq t_{5}$ would be exactly the charging energy $E_{d} / \gamma_{d} \gamma_{c}$ where $\gamma_{c}$ is the efficiency of the PHS charging process. The PHS charging process can then terminate.

In view of the above, thus over the interval $t_{3} \leq t \leq t_{4}$, set

$$
\left.\begin{array}{c}
P_{\mathrm{DESS}, \text { ref }}(t)=P_{L, f}(t)-P_{D, \min } \\
P_{D, \text { ref }}(t)=P_{D, \min }(t)
\end{array}\right\} \text { for } t_{3} \leq t \leq t_{4} .
$$

Starting from $t_{4}, P_{D \text {,ref }}(t)$ is to follow the linear power ramp-up curve shown in Fig. 6(b). Hence,

$$
\left.\begin{array}{c}
P_{\mathrm{DESS}, \text { ref }}(t)=P_{D, \text { ref }}\left(t_{4}\right)-\gamma_{\max }\left(t-t_{4}\right) \\
\left.P_{D, \text { ref }}(t)=P_{L, f}(t)+\gamma_{\max }\left(t-t_{4}\right)\right) \\
-P_{\text {DESS,ref }}\left(t_{4}\right)
\end{array}\right\} \text { for } t_{4} \leq t \leq t_{5} .
$$

After $t_{5}$, all the $P_{L, f}(t)$ is to be exported to the grid. Thus, set

$$
\left.\begin{array}{c}
P_{\mathrm{DESS}, \mathrm{ref}}(t)=0 \\
P_{D, \text { ref }}(t)=P_{L, f}(t)
\end{array}\right\} \text { for } t_{5} \leq t \leq t_{1}+z
$$

The PHS shall then be in the idle state as in (9) and its SOE is precisely at the reference level $\mathrm{SOE}^{*}$. The process of (15) is maintained until the next instance when another Scenario 2 is predicted and the discharging-charging cycle of the PHS is repeated.

In summary, at time $t_{0}$, the dispatch power reference $P_{D, \text { ref }}(t)$ for $t_{1} \leq t \leq t_{1}+z$ is governed by (9)-(15). According to Fig. 6, as fresh $P_{L}(t)$ becomes available at the end of the wind power processing and analysis stage, $P_{L, f}(t)$ can be readily generated by the ANN forecaster within the dispatch schedules re-submission interval $s$. Therefore the proposed dispatch planning scheme would allow the dispatch reference $P_{D \text {,ref }}(t)$ to be updated in time to meet the power market requirements.

Similar analysis can be carried out to determine $P_{D \text {,ref }}(t)$ when a Scenario 3 event of Section IV-A occurs: when $P_{L, f}(t)>P_{D, \max }$, the DESS shall store the surplus power. The details are not elaborated in order to conserve space.

Finally, there would be the inevitable difference between $P_{L}(t)$ and its forecast $P_{L, f}(t)$. From (8), clearly, the PHS does compensate for the difference so that $P_{D \text {,ref }}(t)$ can maintain at the value determined based on $P_{L, f}(t)$. It is the provision of this extra control freedom that increases the robustness 


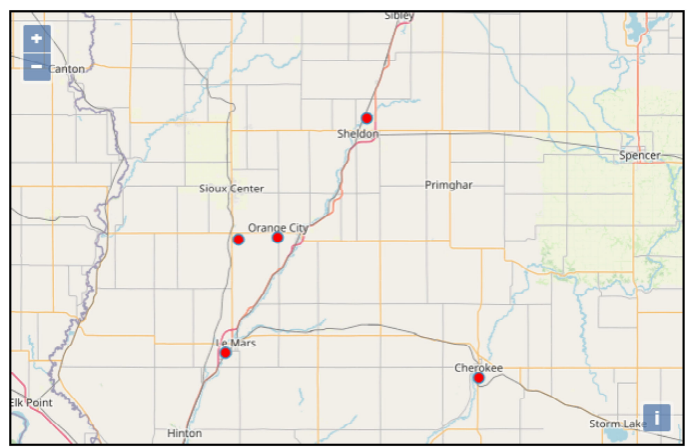

Fig. 7. Geographical locations of the assumed wide-area wind generation scheme under investigation.

of the dispatch plan. Also, the accuracy of $P_{L, f}(t)$ tends to degrade as the time horizon of the forecasts increases. Thus regular update of $P_{L, f}(t)$ is necessary so as to improve on the quality of the dispatch plan $P_{D, \text { ref }}(t)$. It is for this reason that in this investigation and consistent with the re-submission interval $s=1, P_{L, f}(t)$ is updated hourly. $P_{D \text {,ref }}(t)$ beyond the immediate $2 \mathrm{~h}$ will be refreshed once the update in $P_{L, f}(t)$ is available.

\section{ILlUSTRATIVE EXAMPLES}

\section{A. Analysis and Forecasting of Wind Power}

The proposed procedure to dispatch planning for a fictitious 1000-MW wide-area wind power generation scheme is illustrated via numerical examples in this section. WTGs are assumed clustered at the localities of Le Mars $(j=1)$, Orange City $(j=2)$, and Sheldon $(j=3)$ in Iowa, USA. Fig. 7 shows a geographical map of the locations. The clusters are spaced some $20 \mathrm{~km}$ apart and wind speed recorded at these locations can be found in [41]. The recorded wind speed data in 2015 and 2016 in these locations, re-sampled at the rate of $10 \mathrm{~min} / \mathrm{sample}$, was used in this study [42]. The cluster power $P_{W, j}(t)$ was then evaluated using (5), based on the sampled wind speed $v_{W, j}(t)$, and by assuming $v_{W, \text { rated }, j}=12 \mathrm{~m} / \mathrm{s}$, $v_{W, \mathrm{ci}, j}=3 \mathrm{~m} / \mathrm{s}$ and $v_{W, \mathrm{co}, j}=25 \mathrm{~m} / \mathrm{s}$ for the three WTG clusters. A sample of $v_{W, j}(t)$ and $P_{W, j}(t)$ over a selected 30day period is as shown in Figs. 8(a) and (b). Furthermore, it is assumed that $P_{D \text {,min }}=0.1$ p.u., $P_{D \text {, } \max }=1.2$ p.u., and $\gamma_{\max }=0.1$ p.u./min. On $1000 \mathrm{MW}$ power and $1000 \mathrm{MWh}$ energy base, the power and energy storage capacities of the PHS are assumed to be 0.5 p.u. (500 MW) and 1.0 p.u. (1000 MWh) respectively. The maximum depth of discharge $d_{P, \max }$ of the PHS is assumed to be 0.8 and $\mathrm{SOE}^{*}=0.95$.

By applying the procedure of CEEMDAN presented in Section III-B, the IMFs and the residue functions of $P_{W, j}(t)$ were obtained. The number of IMFs $\left(\lambda_{j}\right)$ to be considered as LF-IMFs was evaluated using the method proposed in Section III-D. Depending on the cluster location $j$, it was found that $\lambda_{j}$ can have the value of 6 or 7 when the cycle frequency threshold of $1.0 \times 10^{-4} \mathrm{~Hz}$ was adopted, while the number of IMFs $N_{j}$ is either 9 or 10 . Accordingly, $P_{L, j}(t)$ was next obtained using (7), and Fig. 8(c) shows $P_{L, j}(t)$ over the 30-day period. Next, the area LF wind power component $P_{L}(t)$ was calculated from $P_{L, j}(t)$ using (2). For the purpose
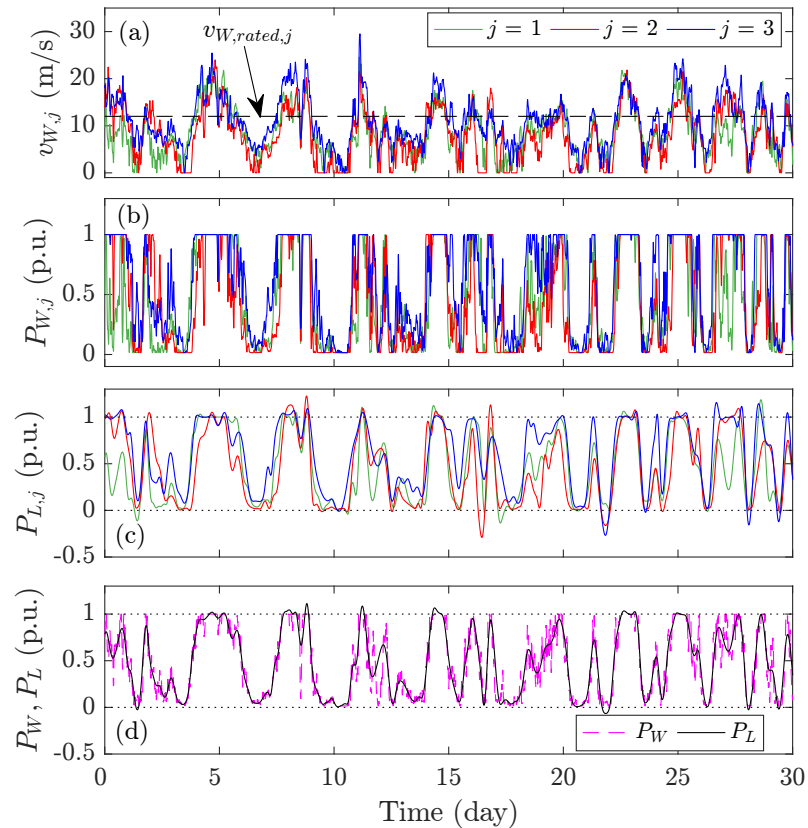

Fig. 8. Example of large-scale wind generation consisting of three WTG clusters: (a) Wind speed. (b) WTG cluster output power expressed on cluster power base. (c) LF component of the cluster output power obtained using CEEMDAN. (d) Area aggregated wind power $P_{W}(t)$ and its low-frequency component $P_{L}(t)$, expressed on area power base.

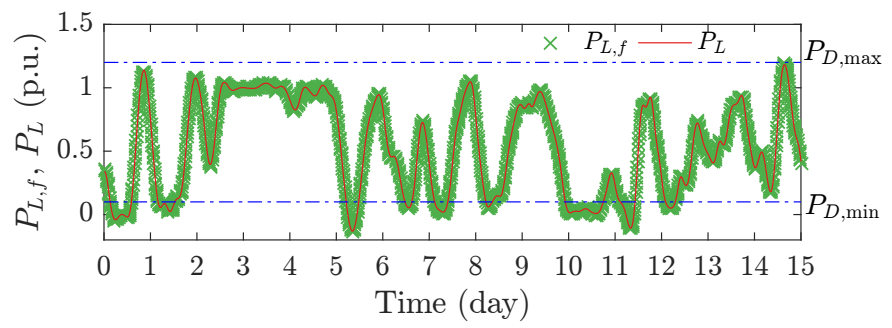

Fig. 9. $P_{L}(t)$ and $P_{L, f}(t)$ over the first 15 days in the second year.

of comparison, Fig. 8(d) shows $P_{L}(t)$ as well as $P_{W}(t)$, the area aggregated wind power obtained by summing the $P_{W, j}(t)$. It can be seen that $P_{L}(t)$ has indeed captured the essence of the LF wind power components. Also, the spatial smoothing effect is apparent when comparing $P_{W, j}(t)$ and $P_{W}(t)$ shown in Fig. 8(c) and Fig. 8(d).

Next, the evaluated $P_{L}(t)$ in the first year was utilized initially to set up and to train a standard back-propagation ANN model which has a single hidden layer and is activated by sigmoid function. As mentioned in Section V-A, although various techniques can meet the need of the proposed framework on LF-IMFs forecast, the ANN technique is well-established and is found to be efficient for the real-time implementation of the framework. Thus, the ANN had been selected to demonstrate the proposed dispatch planning strategy. The input of the ANN includes the first-year $P_{L}$. Design of the ANN model and tuning of the training parameters can be readily done using standard techniques, such as those described in [39], and will not be elaborated.

The model was then used to generate $P_{L, f}(t)$ for the 

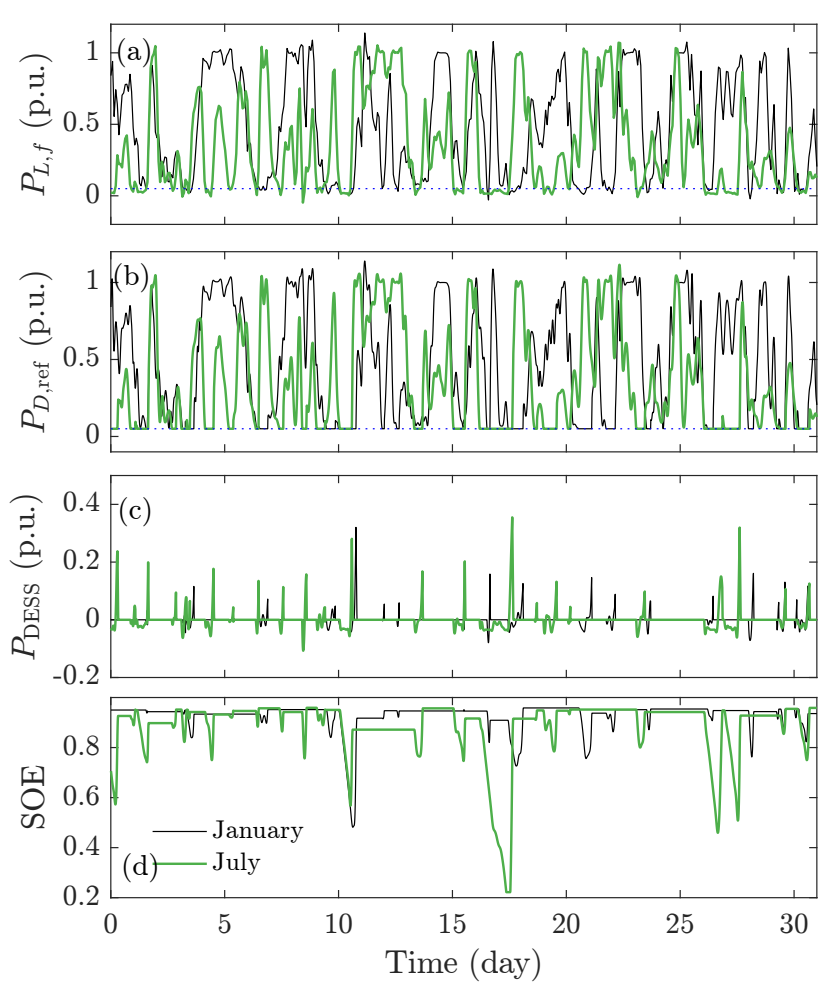

Fig. 10. Examples of the outcome of the dispatch planning of a winter month and of a summer month: (a) Forecasted LF component of the area wind power, (b) Dispatch schedule, (c) PHS power, and (d) SOE of the PHS.

dispatch planning for the first day of the second year. The model and $P_{L, f}(t)$ were next updated hourly, based on the updated $P_{L}(t)$ data of the immediate past 12 months. The performance of the model was also assessed by comparing $P_{L, f}(t)$ with $P_{L}(t)$ of the second year. Fig. 9 shows the comparison for the first 15 days of the second year. As the mean absolute error of the forecast was found to be $1.67 \%$ over the period, it is deemed the ANN model is sufficiently accurate to generate the forecast $P_{L, f}(t)$.

Also over this 15-day period, it can be observed that there are 9 occasions when $P_{L, f}(t)$ is less than the lower dispatch threshold PD,min (Scenario 2 of Section IV-A) and with durations varying from 4.6 to 18.2 hours. On each of these occasions, the DESS is to discharge, in accordance with (11). The discharging/charge intervals are well within the periods in which the PHS can be expected to operate satisfactorily. It verifies in this case the PHS is a suitable DESS medium for the wind power dispatch control task. As $P_{D, \max } \geq P_{L, f}(t)$ over the 15 days, no Scenario 3 event is predicted.

\section{B. Outcome of the Dispatch Planning Process}

The previous subsection shows that ANN can accurately determine the forecast $P_{L, f}(t)$ in the wind power based on the proposed wind power signal analysis and forecasting techniques. The next task is to demonstrate how the dispatch planning for the area wind generation can be carried out. The regularly updated $P_{L, f}(t)$ for a winter month (January) and a summer month (July) of 2016 is shown in Fig. 10(a). By applying the PHS power flow control strategy as described
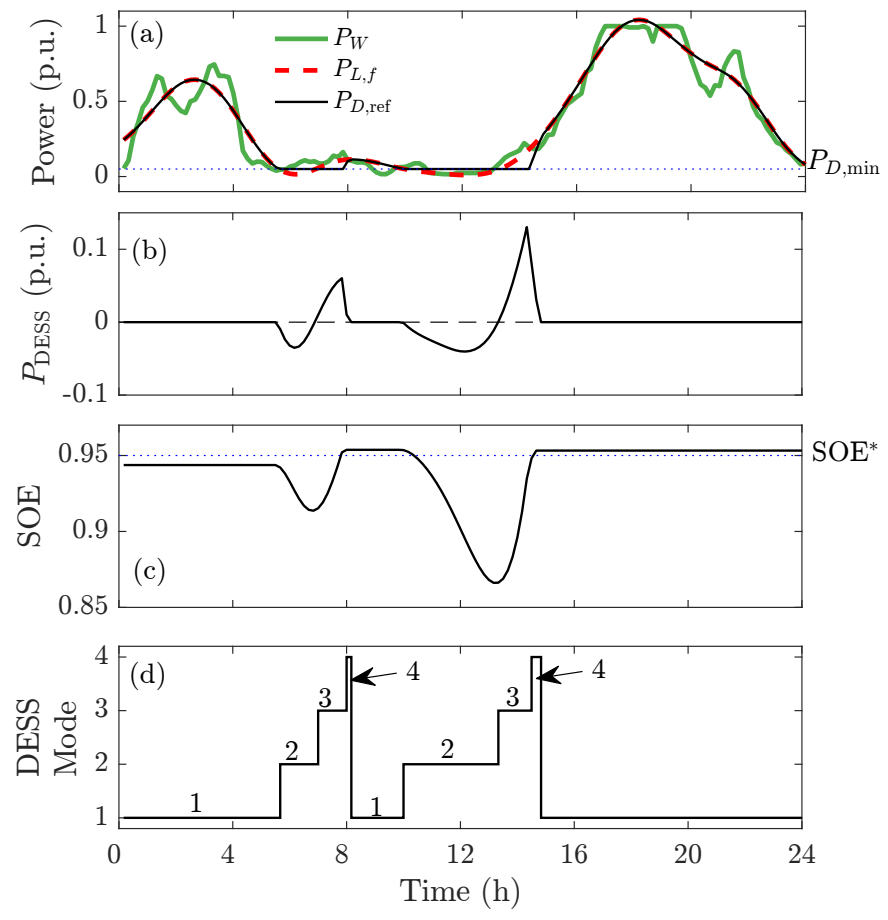

Fig. 11. An expanded view of the dispatch over a 24-h period in late January 2016.

in Section IV-B, the dispatch power reference signal $P_{D \text {,ref }}(t)$ was also dynamically updated and it is as shown in Fig. 10(b). It can be seen that although $P_{L, f}(t)$ does fall below $P_{D \text {,min }}$ on certain intervals, the buffering actions effected by the PHS ensure that $P_{D \text {,ref }}(t) \geq P_{D \text {,min }}$. Using (7), the PHS output power $P_{\mathrm{DESS}}(t)$ was obtained by subtracting $P_{D \text {,ref }}(t)$ from $P_{L, f}(t)$. From Fig. 10(c), it can be seen that the PHS is to undergo some 14 and 19 distinct discharge/charge cycles in January and in July, respectively.

From Fig. 10(b) and Fig. 10(d), it is also observed that although more energy is generated and dispatched to the grid in the winter month, the DESS has to undertake a larger amount of discharging actions in order to achieve the area wind power dispatchability in the summer month. The reason for this is because there are more instances of $P_{L, f}(t)$ dropping below the $P_{D \text {,min }}$ limit of 0.1 p.u. in the summer month (the Scenario 2 events), and the PHS has to discharge to meet the power shortfall. This latter observation is reflected by the larger excursions in the SOE of the PHS in the summer month, as shown in Fig. 10(d). Indeed, the minimum SOE of the PHS is slightly above 0.2 and it occurs around day 17 in that month. This yields the maximum depth of discharge in this month of slightly less than the 0.8 limit assumed in this example. Furthermore, $P_{\mathrm{DESS}}(t)$ over the winter and summer months is well within the assumed PHS power rating of 0.5 p.u. Hence the $500 \mathrm{MW}$ and $1000 \mathrm{MWh}$ PHS power and energy storage capacities are adequate to undertake the buffering task in January and July, 2016.

It would be instructive to examine the expanded view shown in Figs. 11(a) and (b) of $P_{L, f}(t), P_{D \text {,ref }}(t), P_{\text {DESS }}(t)$, and the SOE of the PHS in which two discharging-charging cycles are 
observed. The results of the dispatch planning are pertaining to a selected day in late January 2016. Over the interval indicated as DESS Mode 1 in Fig. 11(d) (correspond to interval $\left[t_{1}, t_{2}\right]$ in Fig. 6), $P_{L, f}(t) \geq P_{D, \text { min }}$ and so $P_{L}(t)$ is to be exported to the grid. Indeed, $P_{D \text {,ref }}(t)$ shows the export is taking place. However, $P_{D}(t)$ is determined based on the forecast $P_{L, f}(t)$ and as shown in Fig. 9, $P_{L, f}(t)$ differs slightly from $P_{L}(t)$. The power difference is buffered by the PHS, although the power flow $P_{\mathrm{DESS}}(t)$ is at a relatively low level and is difficult to decipher from Fig. 11(b). Subsequently over DESS Mode 2 ( $\left[t_{2}, t_{3}\right]$ in Fig. 6), $P_{L}(t) \leq P_{D \text {,min }}$. Based on the dispatch planning strategy (11) and in order to maintain $P_{D \text {,min }}$ for dispatch, the PHS has to discharge to compensate for the mismatch, which leads to the expected decrease of SOE shown in Fig. 11(c).

At about the 7th hour, $P_{L, f}(t)$ is observed to have recovered to a level above $P_{D, \min }$. Instead of exporting all $P_{L, f}(t)$ to the grid, the proposed dispatch planning strategy is to replenish the discharged energy of the PHS so that the re-charged PHS will be ready to provide the buffering actions when the next event of $P_{L}(t)<P_{D, \text { min }}$ or $P_{L}(t)>P_{D, \text { max }}$ were to occur. Accordingly, over the DESS Modes 3 and 4 intervals, the PHS is being charged and its SOE increases $\left(\left[t_{3}, t_{4}\right]\right.$ and $\left[t_{4}, t_{5}\right]$ in Fig. 6), as indicated by its SOE recovering to SOE* After DESS Mode 4, as the PHS SOE is at SOE* and since $P_{L, f}(t)$ is above $P_{D, \min }, P_{L}(t)$ would be exported to the grid in the form of the positive $P_{D \text {,ref }}(t)$.

\section{Comparative Study - Case 1}

In order to validate the efficacy of the proposed dispatch planning strategy, results based on other reported dispatch control strategies are generated and compared with that obtained using the proposed approach. The same set of wind speed data as in Section IV-A was used in these comparative studies. In the first comparative study, a filtering strategy based on the DWT algorithm and similar to that proposed in [21] was used. In essence, the main objective of the strategy in [21] is to ensure the smoothing of the wind power so as to satisfy the wind power injection limits stipulated in grid code. Also, in this comparative study, two sets of optimal wavelets were selected by following the DWT-based design procedure given in [21]. The first and the second half of 2015 historical data were utilized to determine these two sets of the wavelets. The wavelets were next used to design two feedback control strategies (denoted by DWT-1 and DWT2 , respectively) which took into consideration the remaining energy level in the ESS. For each of the strategies, two lowpass filters were next designed to separate the HF and the LF oscillating components. The simulation results for the first 10 days in January 2016 are compared with that obtained using the proposed CEEMDAN-based strategy, as shown in Fig. 12.

Fig. 12(a) shows the reference signals of $P_{D}(t)$ obtained based on the DWT-1, DWT-2 and the proposed approach. Although all the three designs would lead to much smoother dispatch power, nevertheless the differences in the $P_{D}(t)$ do result in the DESS responding in quite dissimilar manner. In the DESS power flows plots shown in Fig. 12(b), the proposed

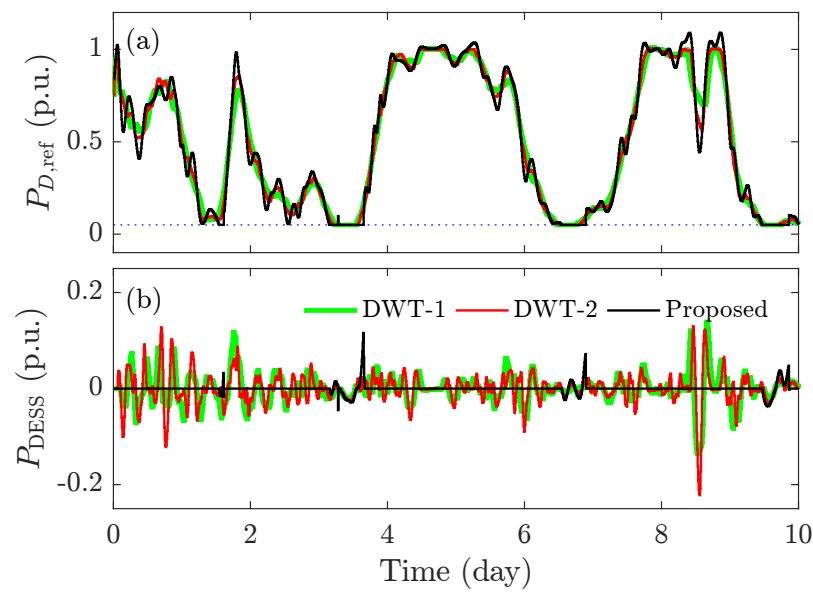

Fig. 12. Comparison of the simulation results based on DWT-filtering and the proposed CEEMDAN-based methods.

approach has resulted in 7 distinct charging/discharging events over the 10 days and the magnitude of the power flows $P_{\mathrm{DESS}}(t)$ is most modest. In comparison, either of the two DWT-based strategies would lead to more than 40 charging/discharging DESS operations over the same period. The resulting $P_{\mathrm{DESS}}(t)$ is also of larger magnitude. Such a relatively large number of operations may be tolerable if BESS or SC ESS is used in the power smoothing task, as in [21]. However, as explained in Section IV-B, in view of their higher capital cost per MWh energy storage capacity, the BESS and SC would be less suitable than the PHS in the present large-scale wind power application. In any case, neither the DWT-1 nor the DWT-2 strategies would be suitable for the PHS. This is because the more frequent and shorter charging/discharging cycle operations shown in Fig. 12(b) would mean that the PHS would be subjected to increased stress. Finally, the example also demonstrates that the two sets of optimal wavelets based on the two different historical data can produce DESS responses which are noticeably different. This adds complexity in the DWT-based design method, a complexity which does not exist in the proposed approach.

\section{Comparative Study - Case 2}

In the next comparative study, the outcome of the dispatch planning based on the proposed procedure is compared with that based on the EMD algorithm and reported in [23]. For the EMD-based dispatch planning approach, the forecasting method and DESS control strategy are identical to those used in the proposed procedure. Based on the same DESS cycle frequency threshold of $1.0 \times 10^{-4} \mathrm{~Hz}$, the EMD-based method yields $N_{j}$ which has the value of 11 or 12 while $\lambda_{j}$ is either 9 or 10. A sample of the LF wind power component as well as the area aggregated wind power over a selected 10-day period in February 2016 is shown in Fig. 13(a). It can be seen that although the two approaches can lead to similar dispatch schedules over many instances, there are distinct differences during periods of high wind. Over these periods, the wind power would be constrained to the area WTG rating (1.0 p.u.). When this occurs, the EMD-based dispatch 

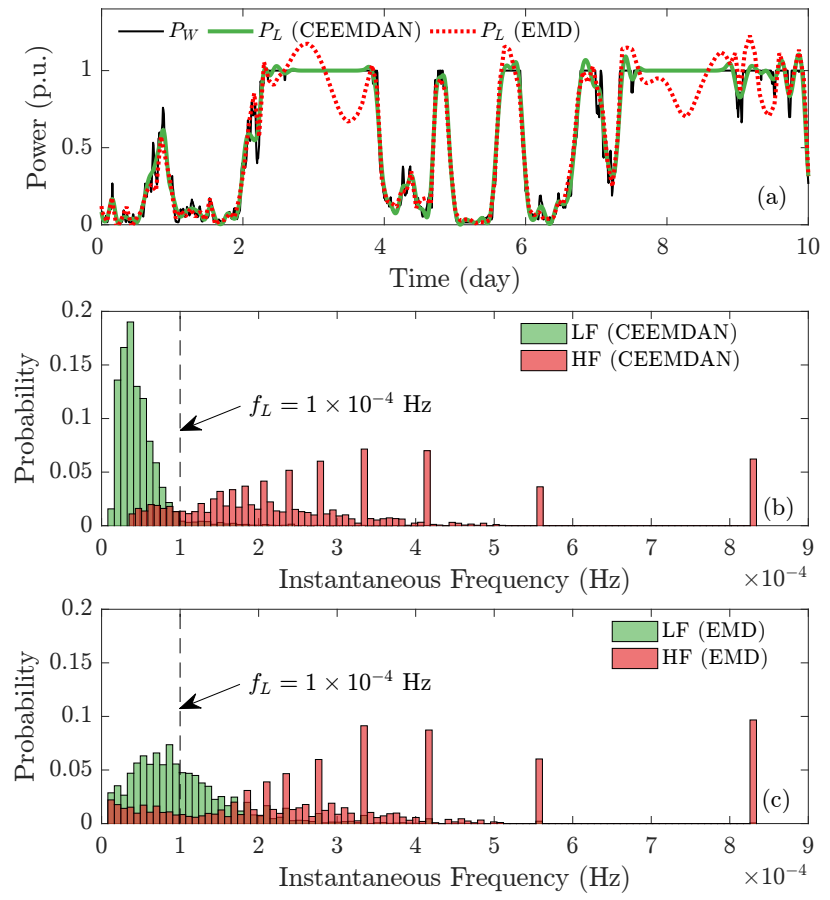

Fig. 13. Comparison of the outcomes of using the CEEMDAN- and EMDbased dispatch planning strategies: (a) Waveforms of the LF wind power component and the aggregated wind power. (b) Probability distributions of the LF and the HF wind power components obtained using the CEEMDAN technique. (c) Probability distributions of the LF and the HF wind power components obtained using the EMD technique.

procedure would introduce large-amplitude LF oscillations. It leads to the difficulty to segregate the LF- and the HF-IMFs, as alluded to in Section III-D, when the EMD technique is used. Indeed, the probability distributions of the instantaneous frequencies of the LF and HF components shown in Fig. 13(c) indicate a considerable region of overlap. The overlap region is much reduced when the proposed approach is used instead, as can be seen in Fig. 13(b).

\section{CONCLUSIONS}

Dispatch planning of wide-area wind generation has been investigated. By applying adaptive CEEMDAN analysis, it is shown that the low-frequency components of the area harnessed wind power can be extracted online. Through the application of the ANN technique, accurate day-ahead forecast of these components can be readily obtained. Based on the forecast, robust short-term day-ahead dispatch schedules of the area wind generation can be obtained in time to satisfy the power market requirements on dispatchability. The dispatch scheduling is also facilitated by controlling the power flows of the centralized DESS which operates in accordance with the developed strategy. Furthermore, by adopting the PHS as the medium of the DESS and by assuming the wind generation has the highest priority on dispatch, it is shown that the PHS can be expected to undertake the energy buffering role occasionally, and the required PHS energy storage capacity is modest in comparison to the amount of the harnessed energy from the wind. While the present work only assumed a centralized
DESS, in the event when multiple DESSs are needed, optimal design and coordination of the buffering actions between these dispatch-oriented ESS would be necessary. This would be a fruitful area for future investigation.

\section{REFERENCES}

[1] IRENA, "Renewable capacity statistics 2020," International Renewable Energy Agency (IRENA), Tech. Rep., Mar. 2020.

[2] T. Ackermann, Wind Power in Power Systems, 2nd ed. Wiley, 2012.

[3] Y. Li, M. Vilathgamuwa, S. S. Choi, B. Xiong, J. Tang, Y. Su, and Y. Wang, "Design of minimum cost degradation-conscious lithium-ion battery energy storage system to achieve renewable power dispatchability," Appl. Energy, vol. 260, p. 114282, Feb. 2020.

[4] "Review of the real-time dispatch algorithm," IESO, Tech. Rep., 2018.

[5] F. Luo, K. Meng, Z. Y. Dong, Y. Zheng, Y. Chen, and K. P. Wong, "Coordinated operational planning for wind farm with battery energy storage system," IEEE Trans. Sustain. Energy, vol. 6, no. 1, pp. 253262, Jan. 2015

[6] Y. Li, S. S. Choi, D. M. Vilathgamuwa, and D. L. Yao, "An improved dispatchable wind turbine generator and dual-battery energy storage system to reduce battery capacity requirement," in Proc. IEEE Southern Power Electron. Conf., Auckland, New Zealand, 5-8 Dec. 2016, pp. 1-6.

[7] J. W. Shim, Y. Cho, S. Kim, S. W. Min, and K. Hur, "Synergistic control of SMES and battery energy storage for enabling dispatchability of renewable energy sources," IEEE Trans. Appl. Supercond., vol. 23, no. 3, p. 5701205, Jun. 2013.

[8] B. S. Pali and S. Vadhera, "An innovative continuous power generation system comprising of wind energy along with pumped-hydro storage and open well," IEEE Trans. Sustain. Energy, vol. 11, no. 1, pp. 145-153, Jan. 2020.

[9] J. Cheng, R. Li, F. F. Choobineh, Q. Hu, and S. Mei, "Dispatchable generation of a novel compressed-air assisted wind turbine and its operation mechanism," IEEE Trans. Sustain. Energy, vol. 10, no. 4, pp. 2201-2210, Oct. 2019.

[10] Z. Chen, Y. Zhang, T. Ji, C. Li, Z. Xu, and Z. Cai, "Economic dispatch model for wind power integrated system considering the dispatchability of power to gas," IET Gener., Transm. Distrib., vol. 13, no. 9, pp. 15351544, May 2019.

[11] K. W. Wee, S. S. Choi, and D. M. Vilathgamuwa, "Design of a least-cost battery-supercapacitor energy storage system for realizing dispatchable wind power," IEEE Trans. Sustain. Energy, vol. 4, no. 3, pp. 786-796, Jul. 2013.

[12] M. Liu, F. L. Quilumba, and W. Lee, "Dispatch scheduling for a wind farm with hybrid energy storage based on wind and LMP forecasting," IEEE Trans. Ind. Appl., vol. 51, no. 3, pp. 1970-1977, May-Jun. 2015.

[13] P. Fairley, "A grid as big as China," IEEE Spectr., vol. 56, no. 3, pp. 36-41, Mar. 2019.

[14] Q. Li, S. S. Choi, Y. Yuan, and D. L. Yao, "On the determination of battery energy storage capacity and short-term power dispatch of a wind farm," IEEE Trans. Sustain. Energy, vol. 2, no. 2, pp. 148-158, Apr. 2011.

[15] X. Han, F. Chen, X. Cui, Y. Li, and X. Li, "A power smoothing control strategy and optimized allocation of battery capacity based on hybrid storage energy technology," Energies, vol. 5, no. 5, May 2012.

[16] X. Li, "Fuzzy adaptive Kalman filter for wind power output smoothing with battery energy storage system," IET Renew. Power Gener., vol. 6 , no. 5, pp. 340-347, Sep. 2012.

[17] A. M. Howlader, H. Matayoshi, and T. Senjyu, "A robust $H_{\infty}$ controller based gain-scheduled approach for the power smoothing of wind turbine generator with a battery energy storage system," Electr. Power Compon. Syst., vol. 43, no. 19, pp. 2156-2167, Nov. 2015.

[18] M. Jannati, S. H. Hosseinian, B. Vahidi, and G. Li, "Mitigation of windfarm power fluctuation by adaptive linear neuron-based power tracking method with flexible learning rate," IET Renew. Power Gener, vol. 8, no. 6, pp. 659-669, Aug. 2014.

[19] M. Jannati, S. H. Hosseinian, B. Vahidi, and G.-J. Li, "ADALINE (ADAptive Linear NEuron)-based coordinated control for wind power fluctuations smoothing with reduced BESS (battery energy storage system) capacity," Energy, vol. 101, pp. 1-8, Apr. 2016.

[20] Y. V. Makarov, P. Du, M. C. W. Kintner-Meyer, C. Jin, and H. F. Illian, "Sizing energy storage to accommodate high penetration of variable energy resources," IEEE Trans. Sustain. Energy, vol. 3, no. 1, pp. 34 40, Jan. 2012. 
[21] Q. Jiang and H. Hong, "Wavelet-based capacity configuration and coordinated control of hybrid energy storage system for smoothing out wind power fluctuations," IEEE Trans. Power Syst., vol. 28, no. 2, pp. 1363-1372, May 2013.

[22] H. Bitaraf, S. Rahman, and M. Pipattanasomporn, "Sizing energy storage to mitigate wind power forecast error impacts by signal processing techniques," IEEE Trans. Sustain. Energy, vol. 6, no. 4, pp. 1457-1465, Jul. 2015.

[23] Y. Yuan, C. Sun, M. Li, S. S. Choi, and Q. Li, "Determination of optimal supercapacitor-lead-acid battery energy storage capacity for smoothing wind power using empirical mode decomposition and neural network," Electr. Power Syst. Res., vol. 127, pp. 323-331, Oct. 2015.

[24] N. E. Huang, Z. Shen, S. R. Long, M. C. Wu, H. H. Shih, Q. Zheng, N.C. Yen, C. C. Tung, and H. H. Liu, "The empirical mode decomposition and the Hilbert spectrum for nonlinear and non-stationary time series analysis," Proc. Roy. Soc. London. Series A: Math. Physical Eng. Sci., vol. 454, no. 1971, pp. 903-995, Mar. 1998.

[25] Z. Wu and N. E. Huang, "Ensemble empirical mode decomposition A noise-assisted data analysis method," Advances in Adaptive Data Analysis, vol. 1, no. 1, pp. 1-41, Jan. 2009.

[26] Y. Ren, P. N. Suganthan, and N. Srikanth, "A comparative study of empirical mode decomposition-based short-term wind speed forecasting methods," IEEE Trans. Sustain. Energy, vol. 6, no. 1, pp. 236-244, Jan. 2015.

[27] N. Bokde, A. Feijo, D. Villanueva, and K. Kulat, "A review on hybrid empirical mode decomposition models for wind speed and wind power prediction," Energies, vol. 12, no. 2, p. 254, Jan. 2019.

[28] M. E. Torres, M. A. Colominas, G. Schlotthauer, and P. Flandrin, "A complete ensemble empirical mode decomposition with adaptive noise," in Proc. IEEE Int. Conf. Acoustics, Speech Signal Process., 22-27 May 2011, pp. 4144-4147.

[29] P. Li, H. Banakar, P. K. Keung, H. G. Far, and B. T. Ooi, "Macromodel of spatial smoothing in wind farms," IEEE Trans. Energy Convers., vol. 22, no. 1, pp. 119-128, Feb. 2007.

[30] I. N. Moghaddam, B. Chowdhury, and M. Doostan, "Optimal sizing and operation of battery energy storage systems connected to wind farms participating in electricity markets," IEEE Trans. Sustain. Energy, vol. 10, no. 3, pp. 1184-1193, Jul. 2019.

[31] T. Jin, F. Zhuo, and M. A. Mohamed, "A novel approach based on CEEMDAN to select the faulty feeder in neutral resonant grounded distribution systems," IEEE Trans. Intrum. Meas., vol. 69, no. 7, pp. 4712-4721, Jul. 2020.

[32] A. Humeau-Heurtier, G. Mahé, and P. Abraham, "Multi-dimensional complete ensemble empirical mode decomposition with adaptive noise applied to laser speckle contrast images," IEEE Trans. Med. Imag., vol. 34, no. 10, pp. 2103-2117, Apr. 2015

[33] W. Lu, Y. Rui, Z. Yi, B. Ran, and Y. Gu, "A hybrid model for lane-level traffic flow forecasting based on complete ensemble empirical mode decomposition and extreme gradient boosting," IEEE Access, vol. 8, pp. 42 042-42 054, Feb. 2020.

[34] P. Mandal, K. Tank, T. Mondal, C. Chen, and M. J. Deen, "Predictive walking-age health analyzer," IEEE J. Biomed. Health Informat., vol. 22, no. 2, pp. 363-374, Mar. 2018

[35] H. Zhao, Q. Wu, S. Hu, H. Xu, and C. N. Rasmussen, "Review of energy storage system for wind power integration support," Appl. Energy, vol. 137, pp. 545-553, Jan. 2015

[36] S. Rehman, L. M. Al-Hadhrami, and M. M. Alam, "Pumped hydro energy storage system: A technological review," Renew. Sustain. Energy Rev., vol. 44, pp. 586-598, Apr. 2015.

[37] M. E. Khodayar, L. Abreu, and M. Shahidehpour, "Transmissionconstrained intrahour coordination of wind and pumped-storage hydro units," IET Gener., Transm. Distrib., vol. 7, no. 7, pp. 755-765, Jul. 2013.
[38] L. Ma, S. Luan, C. Jiang, H. Liu, and Y. Zhang, "A review on the forecasting of wind speed and generated power," Renew. Sustain. Energy Rev., vol. 13, no. 4, pp. 915-920, May 2009.

[39] S. Shanmuganathan and S. Samarasinghe, Artificial Neural Network Modelling. Switzerland: Springer International Publishing, 2016.

[40] "Technical regulation 3.2.5 for wind power plants with a power output greater than $11 \mathrm{~kW}$," Energinet.dk, Tech. Rep., Jun. 2016.

[41] Iowa Environmental Mesonet: AWOS Network Database. [Online]. Available: http://mesonet.agron.iastate.edu/request/download.phtml?network=AWOS

[42] M. Li, Y. Li, and S. S. Choi, "10 minutely wind speed 2015-2016," Sep. 30, 2020. [Online]. Available: https://dx.doi.org/10.21227/tebg-0d02

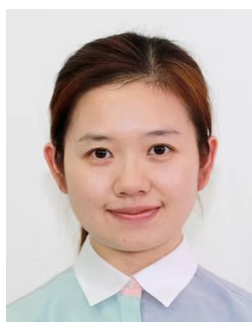

Mengting Li received the B.E. degree in electrical engineering from Chongqing University, Chongqing, China, in 2008 and the Ph.D degree in power engineering from the Nanyang Technological University, Singapore, in 2016

Her research interests include power system control, renewable energy, and energy storage systems.

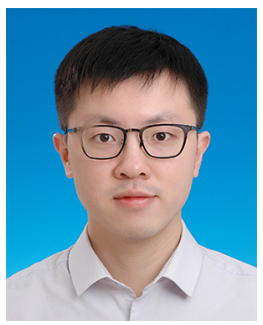

Yang Li (Member, IEEE) received the B.E. degree in electrical engineering from Wuhan University, Wuhan, China, in 2007, and the M.Sc. and Ph.D. degrees in power engineering from the Nanyang Technological University (NTU), Singapore, in 2008 and 2015 , respectively.

From 2015 to 2018, he was a Research Fellow with the Energy Research Institute, NTU and the School of Electrical Engineering and Computer Science, Queensland University of Technology, Brisbane, Australia. Since 2019, he has been with the School of Automation, Wuhan University of Technology, Wuhan, China. $\mathrm{He}$ is currently also a Visiting Professor with the Department of Electrical Engineering, Chalmers University of Technology, Gothenburg, Sweden. His research interests include modeling and control of energy storage systems in power grid and transport sectors.

Dr. $\mathrm{Li}$ is a recipient of the EU Marie Skłodowska-Curie Action Individual Fellowship in 2020 .

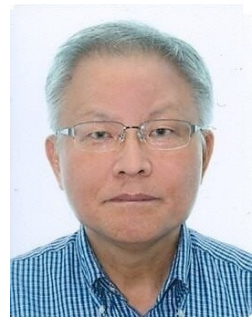

San Shing Choi received the B.E. and Ph.D. degrees in electrical engineering from the University of Canterbury, Christchurch, New Zealand, in 1973 and 1976, respectively. He was previously with the New Zealand Electricity Department, Wellington, New Zealand, National University of Singapore, Singapore, the State Energy Commission of Western Australia, Perth, Australia, and Nanyang Technological University, Singapore. He is currently an Adjunct Professor with the Curtin University of Technology, Perth, and Queensland University of Technology,

Brisbane, both in Australia.

His research interests include power system control, renewable, and energy storage systems. 